\title{
Hipsters on networks: How a minority group of individuals can lead to an antiestablishment majority
}

\author{
Jonas S. Juul* \\ Niels Bohr Institute, University of Copenhagen, Blegdamsvej 17, Copenhagen 2100-DK, Denmark \\ Mason A. Porter ${ }^{\dagger}$ \\ Department of Mathematics, University of California, Los Angeles, California 90095, USA; \\ Oxford Centre for Industrial and Applied Mathematics, Mathematical Institute, University of Oxford, Oxford OX2 6GG, United Kingdom; \\ and CABDyN Complexity Centre, University of Oxford, Oxford OX1 1HP, United Kingdom
}

(Received 28 November 2018; published 26 February 2019)

\begin{abstract}
The spread of opinions, memes, diseases, and "alternative facts" in a population depends both on the details of the spreading process and on the structure of the social and communication networks on which they spread. One feature that can change spreading dynamics substantially is heterogeneous behavior among different types of individuals in a social network. In this paper, we explore how antiestablishment nodes (e.g., hipsters) influence the spreading dynamics of two competing products. We consider a model in which spreading follows a deterministic rule for updating node states (which indicate which product has been adopted) in which an adjustable probability $p_{\text {Hip }}$ of the nodes in a network are hipsters, who choose to adopt the product that they believe is the less popular of the two. The remaining nodes are conformists, who choose which product to adopt by considering which products their immediate neighbors have adopted. We simulate our model on both synthetic and real networks, and we show that the hipsters have a major effect on the final fraction of people who adopt each product: even when only one of the two products exists at the beginning of the simulations, a small fraction of hipsters in a network can still cause the other product to eventually become the more popular one. To account for this behavior, we construct an approximation for the steady-state adoption fractions of the products on $k$-regular trees in the limit of few hipsters. Additionally, our simulations demonstrate that a time delay $\tau$ in the knowledge of the product distribution in a population, as compared to immediate knowledge of product adoption among nearest neighbors, can have a large effect on the final distribution of product adoptions. Using a local-tree approximation, we derive an analytical estimate of the spreading of products and obtain good agreement if a sufficiently small fraction of the population consists of hipsters. In all networks, we find that either of the two products can become the more popular one at steady state, depending on the fraction of hipsters in the network and on the amount of delay in the knowledge of the product distribution. Our simple model and analysis may help shed light on the road to success for antiestablishment choices in elections, as such success-and qualitative differences in final outcomes between competing products, political candidates, and so on-can arise rather generically in our model from a small number of antiestablishment individuals and ordinary processes of social influence on normal individuals.
\end{abstract}

DOI: 10.1103/PhysRevE.99.022313

\section{INTRODUCTION}

The study of spreading phenomena on networks has received considerable attention in many disciplines, including sociology, economics, physics, biology, computer science, mathematics, and others [1-18]. In analogy with the spread of infectious diseases in populations of susceptible individuals, the spread of social phenomena (such as opinions, actions, memes, information, misinformation, and alternative facts) is often viewed as a contagion process that spreads through a network's nodes, which are connected to each other via one or more types of edges. The nodes can represent entities such as people or institutions [19-21] (or other things); and the edges can represent physical proximity, communication chan-

\footnotetext{
*jonas.juul@nbi.ku.dk

${ }^{\dagger}$ mason@math.ucla.edu
}

nels, sociological interactions (e.g., different types of relationships), or something else. An important goal of many studies of the spread of social contagions is the identification of criteria that determine when the phenomenon that is spreading reaches a large fraction of a population or subpopulation [1,2].

Scholars have used various approaches for studying contagions on networks. These include game theory [7], statistical physics [10], agent-based models [22], and systems of coupled differential equations or stochastic processes [2,23-27]. The temporal dynamics and peak size of an outbreak are influenced both by the specific model of a contagion and by the structure of the network on which it spreads $[12,20,25,27-$ 34]. A key idea is to examine when a disease or idea-perhaps one that initially is present in a small fraction of nodes-can become widespread in a network. When a large fraction of a population or subpopulation becomes infected (or adopts an idea), one says that a cascade has occurred. Cascading phenomena have been studied in a wide variety of systems, 
ranging from financial networks [21] to social media like Twitter [35]. For example, a failing financial institution can cause a cascade of failures of numerous other financial institutions, a tweet can result in a cascade of tweets that promotes the opinion of the original tweeter (perhaps influenced by the actions of "bot" or sockpuppet accounts [17]), and widely spread alternative facts can influence the opinions of a large population of voters [36]. Notwithstanding these dystopian examples, cascading behavior can be beneficial, neutral, or harmful.

Models of cascading behavior on networks can have either stochastic or deterministic state-update rules, and the update rules in most models only consider nodes that are adjacent to a focal node. One example of models that traditionally have deterministic update rules is threshold models for social contagions. The simplest example is the Watts threshold model (WTM) [5,6,9], a type of bootstrap percolation [37], in which each node is assigned a threshold from some distribution. When considering a node for updating, if the fraction of its neighbors that are adopters is at least as large as its threshold, it too becomes an adopter. There are numerous variants and generalizations of the WTM, including ones with adoption thresholds that are based on the number (rather than the fraction) of neighbors who are adopters [12,38], ones with multiple adoption stages [25], ones with "synergy" from other nearby adopters [27], and ones with timers in addition to adoption thresholds [39].

Efforts to develop mathematical models for the spread of products or innovations date at least as far back as the 1960s. Rogers [40] gave a qualitative description (as a sigmoidal shape) of the number of adopters as a function of time. Bass [41] developed a model for the adoption of innovations that was inspired by models for biological contagions. Bass's model results in sigmoidal-shaped adoption curves, and it has been generalized in various ways [42-45]. More recent studies have considered models in which agents of different types can have significant effects on the final distributions of products or innovations in a population. For example, Gordon et al. [46] showed that temporal cycles of adoption can occur if some nodes are allowed to regret adopting an innovation while other ("contrarian") nodes resist adopting innovations. References $[47,48]$ found rich behavior (including chaotic dynamics) in a social contagion model that incorporates an aversion to complete conformity.

Contrarian agents, a key aspect of the present paper, have been incorporated into various types of models of opinion dynamics and hierarchy formation. In the 1980s, Galam [49] illustrated a hierarchical mechanism that allows a minority community to elect its preferred candidate instead of that of the majority. Galam and collaborators have also examined the effects of contrarian [50] and stubborn [51,52] agents on opinion dynamics (though typically without any network structure). Nyczka et al. [53] and Nyczka and Sznajd-Weron [54] studied various opinion models (e.g., $q$-voter models) on a complete graph to highlight an important distinction between two types of nonconformity-anticonformity and independence - that have distinct implications for social dynamics. Khalil and Toral [55] incorporated contrarians into a noisy voter model, and they illustrated that a few contrarians can substantially alter the dynamics of the model.
Apriasz et al. [56] examined an opinion model that includes "snobs," who conform to nodes in their own community but anticonform to nodes in others, to examine how the density of connections between two communities can affect phenomena such as fashion cycles. One can also consider contrarian individuals in the context of economic markets, such as in work by Sznajd-Weron and Weron [57], who studied an Ising model on a rectangular lattice to model advertising in duopoly markets. More recent work related to contrarian agents, in addition to [46-48], includes that of Mellor et al. [58], who examined a population in which nodes can either adopt a product or become "luddites," who oppose the spread of innovation. They found that luddites greatly limit adoption if the adoption rate is high but not if it is low. Gambaro and Crokidakis [59] illustrated that contrarian agents can be a source of disorder in opinion dynamics, and Ferrara and collaborators have investigated how individual social-media accounts controlled by bots can exert a considerable influence on political elections and social cascades [17,60,61].

Anticonformity can manifest in a variety of ways in society. For example, it has been reported that partisan bias can result in some groups of individuals misinterpreting data and explanations of experts (e.g., with respect to the issue of climate change), in conflict with the intended message, such that it fits with the personal beliefs of the group [62,63]. In a recent example about information spreading, Petersen et al. [64] provided psychological assessment of motivations to share hostile political rumors (e.g., in the form of "fake news") among citizens of democratic societies, concluding that such rumors are often shared by individuals because they believe it can mobilize their audience against a disliked establishment (rather than because they think that these rumors are true).

In the present paper, we examine the influence of antiestablishment nodes, such as hipsters, on spreading processes in a social network. Individuals who specifically prefer something other than the established standard in society have manifested in several ways over the last decade. They include members of antiestablishment movements in Western Europe and the United States of America, who have hugely impacted the geopolitical landscape, to the curious style of hipsters in cities throughout the world. In some cases, such as the 2016 "Brexit" vote [65] and the 2016 American presidential election [66], antiestablishment opinions appear to have spread to so many people that they exerted a major influence on political outcomes. In this paper, we ask the following questions: (1) How does a large fraction of a population decide to choose something different from the established standard? (2) How can a small fraction of individuals spread their antiestablishment opinions to a majority (or at least to a very large minority) of the rest of a population? (3) Can we capture these ideas using a simple mathematical model of a spreading process on a network?

A few years ago, a statistical-physics approach was used to examine how anticonformists (i.e., hipsters) who make decisions that differ from that of a majority, perhaps in an attempt to stand out from the crowd, may all end up "looking the same" [67] (wearing the same clothes, buying the same products, having the same opinions, and so on). This study observed that the dynamics of a population was influenced greatly by delays in the knowledge of hipsters and by how 


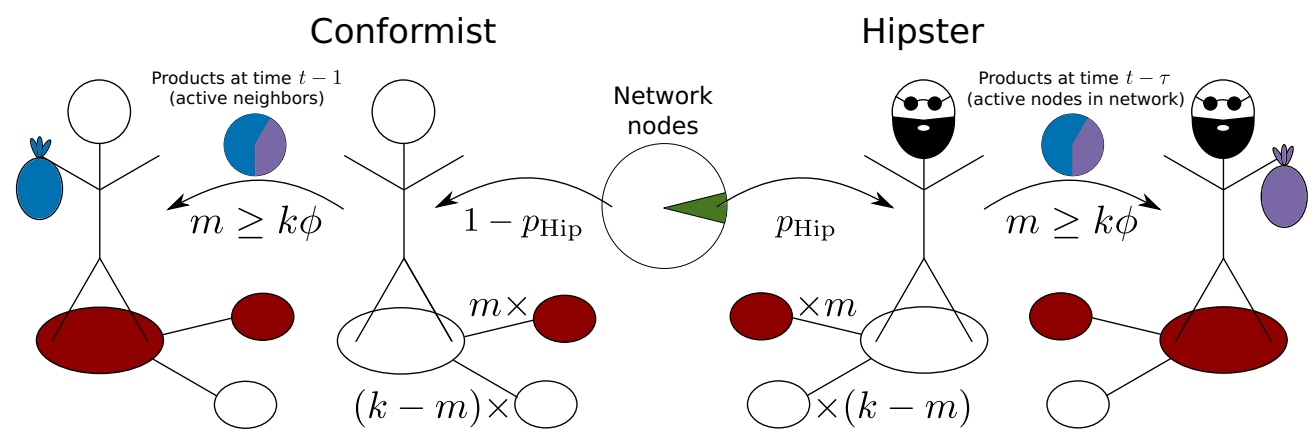

FIG. 1. Illustration of our model of a threshold-based social contagion with hipsters. A node is a hipster with probability $p_{\text {Hip }}$, and it is a conformist with probability $1-p_{\text {Hip }}$. If at least a fraction $\phi_{i}$ of the neighbors of node $i$ are active (as indicated by the red coloring) at discrete time step $t-1$, the node activates and adopts a product at time step $t$ (for $t \geqslant 1$ ). We then need to consider which products have been adopted by node $i$ 's neighbors and the relative popularity of different products in the whole network. If node $i$ is a conformist, it adopts the product that is more popular among its active neighbors at time $t-1$. However, if node $i$ is a hipster, it adopts the product that is less popular among the active nodes in the network at time step $t-\tau$ (where $\tau \in \mathbb{N}$ ). For both node types, a tie results in a node choosing one of the two products (blue versus purple) with equal probability.

large a fraction of the population are hipsters. In the model in [67], individuals interact with their environments and switch between two states with a probability that depends on this environment and on whether an individual is a conformist (preferring to be aligned with its environment) or a hipster (preferring to be opposite to its environment). The model has a phase transition that determines whether or not hipsters ultimately attain the same state. Touboul [67] referred to the anticonformists with delayed knowledge as "hipsters." Because the model that we introduce in this study includes anticonformists with delayed knowledge of the global product distribution, we adopt this terminology. However, our approach, focus, and type of model-which builds on threshold models for social contagions - are rather different from those in [67].

We will explore how anticonformists (i.e., hipsters) affect the spreading of competing products in a network by generalizing the WTM to a network with two types of nodeshipsters and conformists-who respond differently to adoptions. Conformists prefer to adopt the product (or meme, opinion, message, etc.) that is more popular among their active neighbors at time $t-1$. Hipsters, however, prefer to adopt the product that is the less popular of the two products in the whole network at some previous time $t-\tau$. Their choice to adopt a product uses the same adoption condition as that of the conformists. This is a strong assumption, and we make it partly for simplicity (as it allows us to build from the WTM) and partly because it reflects a scenario in which an anticonformist may more actively rebel on an issue that is sufficiently established in its neighborhood in a network. In our model, both conformists and hipsters first choose to buy some product or form an opinion, and then they choose which one to adopt. In their study of the effect of luddites, Mellor et al. [58] assumed that the probability of a node becoming a luddite is proportional to the rate of change in the density of adopters of its neighbors. This resembles our choice that a node's neighborhood influences whether or not it elects to adopt a product, but it differs from our choice that each of our nodes is either inherently a conformist or inherently a hipster. The delay $\tau$ in our model encodes the fact that knowledge about the total population is not instantly available; instead, it is collected over some time before it is available. See Gleeson et al. [68] for a model (without network structure) that illustrates another type of competition between local information (in the form of a social-media feed) and global information (in the form of a bestseller list).

The rest of our paper is organized as follows. In Sec. II, we introduce our model for the spreading of two competing products on networks under the influence of hipster nodes. In Sec. III, we examine our model on a Facebook network. In Sec. IV, we develop an analytical approximation to describe the time-dependent fractions of nodes that adopt the products (where each adopter chooses one of the two competing products) as a function of their degree $k$ and an adoption threshold $\phi$. In Sec. V, we examine our model on several classes of empirical networks and investigate the final fractions of nodes that adopt the products as a function of the time delay $\tau$ and the hipster probability $p_{\text {Hip. }}$. In Sec. VI, we explain the observed behavior in the limit of few hipsters, and we obtain an approximation for the fraction of nodes that adopt the products as a function of the number of hipsters. We conclude and discuss our results in Sec. VII.

\section{A THRESHOLD MODEL WITH HIPSTERS}

Threshold models of social influence are a popular type of spreading process to study on networks $[1,2,6]$. To set up a simple example of a threshold model, consider a network with $N$ nodes, and suppose that each node $i$ has an independently assigned threshold $\phi_{i}$ that we draw from a distribution $f(\phi)$. We also suppose that a node can be in one of two states: active or inactive. An active node has adopted a product (or meme, opinion, etc.) that is spreading in a population, and an inactive node has not adopted the product. (We will use the term "product" from now on.) Once a node becomes active, it stays active forever. The threshold of a node determines how difficult it is to activate that node, so one can construe a node's threshold value as its stubbornness level. Node $i$ becomes active if a peer pressure, which in the WTM is equal to the fraction of active nodes among $i$ 's neighbors, is greater than or equal to its threshold $\phi_{i}$.

We seek to develop a model for competing products that spread in a population that includes hipsters. Therefore, in our 
model, each node $i$ has a value $H_{i} \in\{0,1\}$, such that $H_{i}=$ 0 indicates that node $i$ is a conformist and $H_{i}=1$ indicates that node $i$ is a hipster. We update nodes synchronously. At each discrete time $t \geqslant 1$, we assume that conformists know the distribution of products among their immediate neighbors at the previous time step $t^{\prime}=t-1$, whereas hipsters know the distribution of products in the total population at an earlier time step $t_{\tau}=t-\tau$ (where $\tau \in \mathbb{N}$ ). The first updating step occurs at $t=1$. If $t-\tau<0$, we let $t_{\tau}=0$.

A node chooses to adopt a specific product in two steps. First, the node must become active, which occurs if sufficiently many of its neighbors are active. If the fraction of neighbors that are active at time $t-1$ is at least as large as the node's threshold, it becomes active at time $t$. If node $i$ becomes active, it immediately adopts one of two possible products. If $H_{i}=0$, node $i$ is a conformist and thus adopts the product that is more popular among its active neighbors at time step $t-1$. However, if $H_{i}=1$, node $i$ is a hipster and thus adopts the product that is less popular in the total population at time $t_{\tau}=t-\tau$. For both values of $H_{i}$, a tie results in the node choosing one of the two products with equal probability. Each node can adopt only a single product, and once it has adopted a product, it never switches to the other product or becomes inactive. To keep track of the product distribution, we associate a variable $S_{i}$ with each node $i$. If $S_{i}=0$, node $i$ is inactive; if $S_{i}=1$, node $i$ has adopted product $A$; and if $S_{i}=2$, node $i$ has adopted product $B$. We summarize our model and the decision process in Fig. 1 and its caption. See [79] for a PYTHON script to simulate our model.

At $t=0$, we activate a single node with product $A$, and we introduce product $B$ when the first hipster chooses to adopt a product. In principle, it is possible to generalize our model to consider arbitrarily many products spreading on a network, but we consider only the case of two products for simplicity.

Although it may seem somewhat artificial that the conformists in our model use only local information to decide which product to adopt, whereas the hipsters use only global information, it is both convenient and illustrative (because the WTM has been studied so meticulously) to generalize the WTM model by adding one specific feature. This is also appropriate for exploring the competition between local and global forms of influence. We examine our hipster model both on synthetic networks and on empirical social networks. Our main goal is to examine whether (and when) a small probability $p_{\text {Hip }}$ of hipster nodes can lead to a majority of a network's nodes adopting a product that is less popular than another product at the beginning of a spreading process. We find that the fraction of nodes that adopt the less popular of our two products depends in an interesting way on the delay $\tau$ in the hipsters' knowledge of the product distribution in the total population.

\section{SIMULATION OF OUR MODEL ON A FACEBOOK NETWORK}

We start by simulating our model on the NORTHWESTERN25 network from the FACEBOOK100 data set [69]. This network consists of the friendship relationships on Facebook at Northwestern University on one day in autumn 2005. The network has 10537 nodes, a mean degree of $\langle k\rangle \approx 92$, and
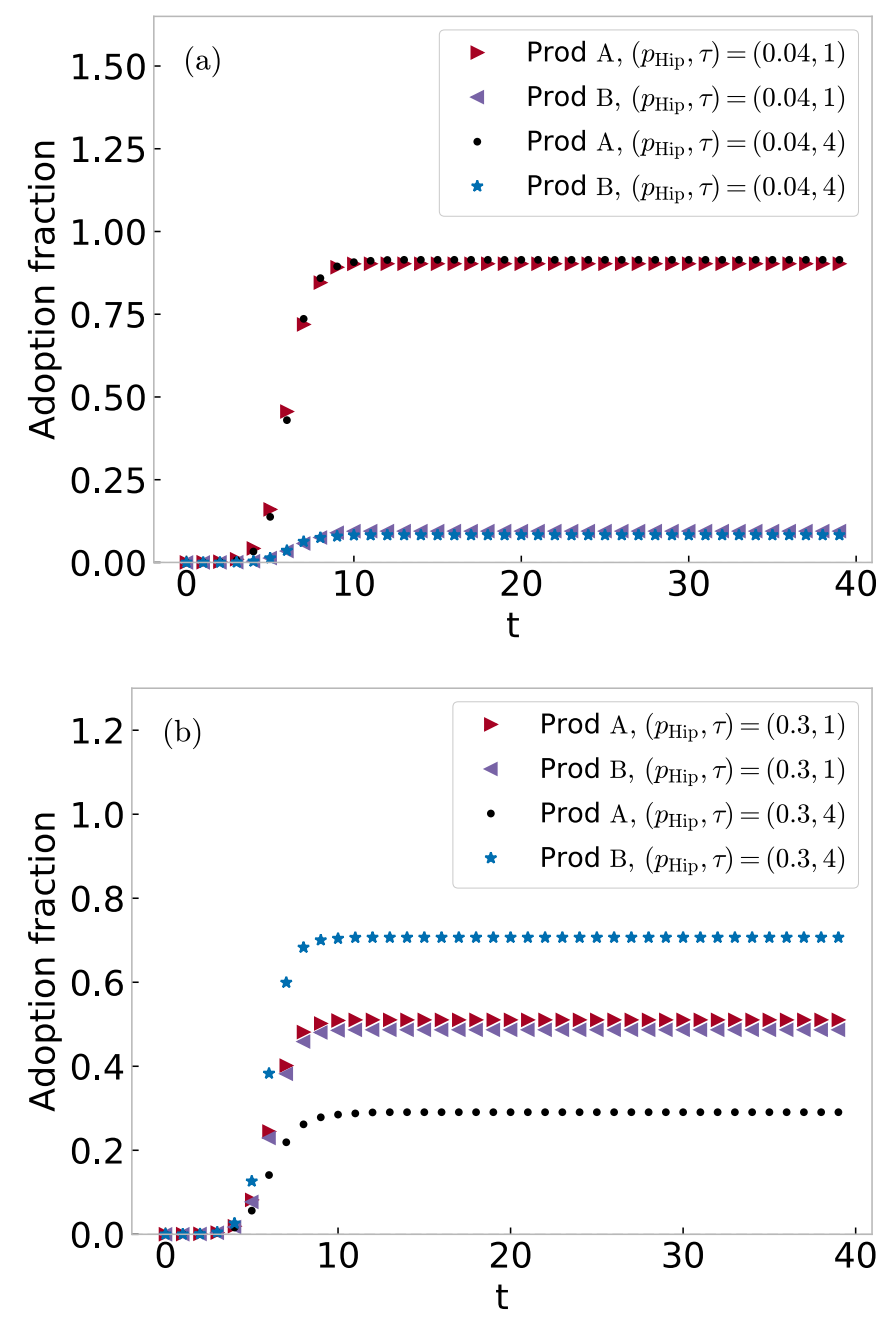

FIG. 2. Example of the behavior of our hipster threshold model on a Facebook network for delay values $\tau=\{1,4\}$ for (a) a hipster probability of $p_{\text {Hip }}=0.04$ and (b) a hipster probability of $p_{\text {Hip }}=$ 0.30 . In each panel, we show the fraction of nodes in the network that are adopters of each of the two products as a function of time. In panel (a), the curves from the two different values of $\tau$ are almost indistinguishable from each other. In panel (b), the adoption fractions of the two products are clearly different when we use different delays ( $\tau=1$ and $\tau=4$ ). For $\tau=1$, the final fractions that are adopters of products $A$ and $B$ are approximately equal. However, for $\tau=4$, product $B$ becomes more popular than product $A$. For both panels, each data point is a mean over 200 simulations on the same network (the NORTHWESTERN25 network of the FACEBOOK100 data set [69]), where we choose seed nodes and hipster nodes uniformly at random for each of the simulations.

a maximum degree of $k_{\max }=2105$. We assign a threshold of $\phi=1 / 33$ to each node. In addition to this threshold, we independently assign each node a value $H_{i} \in\{0,1\}$ with some hipster probability $p_{\text {Hip }}$ to be $H_{i}=1$. Therefore, different simulations of our model with a specified hipster probability do not in general have the same number of hipster nodes. We examine our model with two different time delays and two different values for the probability of hipsters in the network. We consider $\tau=\{1,4\}$ and $p_{\text {Hip }}=\{0.04,0.30\}$, and we conduct simulations for the four combinations of these parameter choices. 
For each parameter pair, we choose a single node uniformly at random and suppose that it has adopted product $A$ at time $t=0$. This node acts as a seed for the spreading process on the network. We introduce another product, labeled $B$, when the first hipster node is activated. Thus, product $B$ will never be adopted by any node if the network has no hipsters, and product $A$ has a head start when product $B$ is adopted for the first time. We stop our simulations after the dynamics reaches a steady state, in which no further adoptions occur. At each time step, we track the fraction of the nodes that are adopters of each of the two products. We conduct 200 simulations - each with a seed chosen uniformly at random, with new hipster nodes for each simulation — and, at each time step $t$, we average the fraction of nodes that are adopters of each product over these 200 simulations. We show the results of these simulations in Fig. 2.

In Fig. 2(a), we plot the fraction of nodes in the adopted state for each of the products at time $t$ for simulations in which the hipster probability is 0.04 . For these parameters, the curves are indistinguishable for the two values of the delay time $\tau$. A much larger fraction of nodes adopts product $A$ than product $B$. In Fig. 2(b), we show the corresponding curves for simulations in which the hipster probability is 0.30 . The results for different delay times $\tau$ are now clearly distinguishable. For $\tau=1$, the fraction of nodes that have adopted the two products are approximately equal; for $\tau=4$, however, the fraction of nodes that have adopted product $B$ is much larger than the fraction that have adopted product $A$.

\section{ANALYSIS}

We approximate the temporal spreading of products on a network using a pair approximation (as in $[25,27,32,70]$ ) that relies on the hypothesis that the network is locally treelike [71,72]. Let $\rho_{\lambda}^{(\phi, k)}(t)$ denote the density of nodes with threshold $\phi$ and degree $k$ that are in the adopted state, for a product $\lambda \in\{A, B\}$, at time step $t$. We write the recursion relation

$$
\begin{aligned}
\rho_{\lambda}^{(\phi, k)}(t+1)= & \rho_{\lambda}^{(\phi, k)}(t)+\left[1-\rho_{\lambda}^{(\phi, k)}(t)\right] \sum_{k^{\prime}=1}^{k} F\left(k, k^{\prime}, \phi\right) B_{k^{\prime}}^{k}\left(\sum_{\beta \in\{A, B\}} \bar{q}_{\beta}^{(\phi, k)}(t)\right) \\
& \times\left[\left(1-p_{\text {Hip }}\right) \sum_{k^{\prime \prime}=1}^{k^{\prime}} \Theta\left(k^{\prime \prime}-\frac{k^{\prime}}{2}\right) B_{k^{\prime \prime}}^{k^{\prime}}\left(\frac{\bar{q}_{\lambda}^{(\phi, k)}(t)}{\sum_{\beta \in\{A, B\}} \bar{q}_{\beta}^{(\phi, k)}(t)}\right)\right. \\
& \left.+p_{\text {Hip }} \prod_{\beta \neq \lambda} \Theta\left(\sum_{k, \phi} \rho_{\lambda}^{(\phi, k)}(\max \{t-\tau, 0\})-\sum_{k, \phi} \rho_{\beta}^{(\phi, k)}(\max \{t-\tau, 0\})\right)\right],
\end{aligned}
$$

where $\bar{q}_{\beta}^{(\phi, k)}(t)$ is the probability that a neighbor, chosen uniformly at random, of an inactive node with threshold $\phi$ and degree $k$ is active and has adopted product $\beta \in\{A, B\}$; the "response function" $F\left(k, k^{\prime}, \phi\right)=1$ if $k^{\prime} / k \geqslant \phi$ and $F\left(k, k^{\prime}, \phi\right)=0$ otherwise; $\Theta(x)$ is the step function (it equals 1 for $x>0$, it equals $1 / 2$ for $x=0$, and it equals 0 otherwise); and

$$
B_{l}^{k}(p)=\left(\begin{array}{l}
k \\
l
\end{array}\right) p^{l}(1-p)^{k-l}
$$

is the binomial function for probability $p$. The product over $\beta \neq \lambda$ in Eq. (1) is in fact a product over a single value (so we did not need to use the product symbol), but one must take a product over all values of $\beta$ except for $\lambda$ in a model with three or more competing products. In this more general case, it is also necessary to take the sums over $\beta$ over a larger set of products (and to replace the step function in the product with a more complicated function).

We write $\bar{q}_{k}^{(\phi, k)}(t)$ as a function of $q_{i}^{\left(\phi^{\prime}, k^{\prime}\right)}(t)$, the probability that, for a given inactive node, a neighbor with degree $k^{\prime}$ and threshold $\phi^{\prime}$ is active at time step $t$. This probability is given by

$$
\bar{q}_{\lambda}^{(\phi, k)}(t)=\frac{\sum_{k^{\prime}, \phi^{\prime}} P\left((k, \phi),\left(k^{\prime}, \phi^{\prime}\right)\right) q_{\lambda}^{\phi^{\prime}, k}(t)}{\sum_{k^{\prime}, \phi^{\prime}} P\left((k, \phi),\left(k^{\prime}, \phi^{\prime}\right)\right)},
$$

where $P\left((k, \phi),\left(k^{\prime}, \phi^{\prime}\right)\right)$ is the probability that a node with degree $k$ and threshold $\phi$ is adjacent to a node with degree $k^{\prime}$ and threshold $\phi^{\prime}$. Given an active node, the probability that a particular neighbor with degree $k$ and threshold $\phi$ is active is

$$
\begin{aligned}
q_{\lambda}^{(\phi, k)}(t+1)= & \rho_{\lambda}^{(\phi, k)}(t)+\left[1-\rho_{\lambda}^{(\phi, k)}(t)\right] \sum_{k^{\prime}=1}^{k-1} F\left(k, k^{\prime}, \phi\right) B_{k^{\prime}}^{k}\left(\sum_{\beta \in\{A, B\}} \bar{q}_{\beta}^{(\phi, k)}(t)\right) \\
& \times\left[\left(1-p_{\text {Hip }}\right) \sum_{k^{\prime \prime}=1}^{k^{\prime}} \Theta\left(k^{\prime \prime}-\frac{k^{\prime}}{2}\right) B_{k^{\prime \prime}}^{k^{\prime}}\left(\frac{\bar{q}_{\lambda}^{(\phi, k)}(t)}{\sum_{\beta \in\{A, B\}} \bar{q}_{\beta}^{(\phi, k)}(t)}\right)\right. \\
& \left.+p_{\text {Hip }} \prod_{\beta \neq \lambda} \Theta\left(\sum_{k, \phi} \rho_{\lambda}^{(\phi, k)}(\max \{t-\tau, 0\})-\sum_{k, \phi} \rho_{\beta}^{(\phi, k)}(\max \{t-\tau, 0\})\right)\right] .
\end{aligned}
$$


TABLE I. Summary statistics of the discarded realizations of our hipster threshold model on each network family (or individual network, for NORTHWESTERN25). The second column gives the total number of discarded realizations. In it, we sum the instances from all parameter values, because the values of $\tau$ and $p_{\text {Hip }}$ do not influence the fraction of nodes that activate in a given realization. We show the mean fraction of nodes that are active at steady state for discarded realizations and the standard deviation of this mean. For all networks, the mean fraction of active nodes is much smaller than the threshold fraction of 0.10 , below which we discard realizations. For each choice of parameter values and network, we keep 200 realizations for our samples.

\begin{tabular}{lccc}
\hline \hline Network & Number of discarded realizations & Mean & Standard deviation of the mean \\
\hline 5-regular configuration model & 36 & 0.0001 & 0.0000 \\
3-regular configuration model & 1843 & 0.0002 & 0.0001 \\
Erdős-Rényi $[G(N, p)]$ & 52214 & 0.0072 & 0.0010 \\
NORTHWESTERN25 & 35171 & 0.0001 & 0.0001 \\
\hline \hline
\end{tabular}

The only difference between Eq. (4) and Eq. (1) stems from the following: in Eq. (1), we consider any degree- $k$ node; however, in Eq. (4), we consider a degree- $k$ neighbor of an inactive node. The latter has a maximum of $k-1$ active neighbors, which is therefore the maximum value of the index of the first sum in Eq. (4). In these equations, we have assumed that each neighbor of node $i$ is independent of the others, so we are assuming that this process is occurring on a locally treelike network [2,71]. However, the Facebook network that we used in Sec. III has a large local clustering coefficient [69], so it is not locally treelike.

\section{HIPSTER THRESHOLD MODEL ON SYNTHETIC NETWORKS}

We now test our analytical approximations of Sec. IV by simulating our model on various synthetic networks with $N=10000$ nodes. We assign each node $i$ a threshold $\phi_{i}$ from some probability distribution $f(\phi)$, which we specify in the following subsections. We also independently assign each node $i$ a value $H_{i} \in\{0,1\}$ to determine if it is a hipster. As before, $p_{\text {Hip }}$ denotes the probability of being assigned the hipster value $H_{i}=1$.

As with our simulations on the Facebook network in Sec. III, we select a single node uniformly at random to have adopted product $A$ at time $t=0$. This node is the seed of the spreading process. There is a risk that the chosen seed node is located in a neighborhood of very few vulnerable nodes. (A node that can be activated by a single active neighbor is known as a "vulnerable" node [9].) With such a seed, few nodes are activated in that realization of the dynamical process, and we do not observe a cascade of adoptions (in which many nodes adopt a product).

To focus on situations in which many nodes adopt (either of the products), we consider only realizations in which at least some minimal fraction of nodes eventually adopt a product. We take this minimal threshold to be 0.10 . (Another way to examine situations with a lot of spreading is through "cluster seeding" [29], in which one considers initial conditions in which some node and all of its neighbors start out as adopters.) In Table I, we indicate the number of discarded realizations, the mean fraction of adopting nodes in these simulations, and the standard deviation of this number of adopters for several types of networks. The threshold 0.10 is much larger than the mean fraction of adopters in discarded realizations, and it is much smaller than the fraction of adopting nodes in realizations that we keep. Therefore, this choice of threshold entails a clear separation between realizations with cascades of adoption and those without such cascades. In many of our networks, the number of discarded simulations (in which there are few adoptions) is very large, consistent with the empirical study of Goel et al. [73].

\section{A. 5-regular configuration-model networks}

We consider configuration-model networks [74] in which every node has degree 5. As described in [74,75], we match stubs (i.e., ends of edges) uniformly at random. We suppose that each node has a threshold of $\phi_{i}=0.19$ with probability $p_{0}=0.8$ and a threshold of $\phi_{i}=0.8$ with probability $1-p_{0}=0.2$. Therefore, on average, $80 \%$ of the nodes are vulnerable, and $20 \%$ of the nodes can adopt only when 4 or more of their nearest neighbors are adopters. We select these parameter values because this choice entails that some nodes are vulnerable, whereas other nodes need to have two or more active neighbors to adopt a product; and it ensures that a cascade of product adoptions occurs in most of our simulations.

We examine our hipster threshold model on the networks for time delays $\tau \in\{1,2,3,4,5,6\}$ and hipster probabilities of $p_{\text {Hip }} \in[0,1]$ (in increments of 0.01 ). For each parameter pair $\left(\tau, p_{\text {Hip }}\right)$, we simulate our model on 200 different networks. We independently draw the specific sets of networks for different parameter values, so in general they are not the same networks. For each realization, we stop the simulations after the distribution of product adoptions reaches a steady state, and we track the adoption fractions of the two competing products. From these values, we calculate the mean fraction of nodes that adopt each product over the 200 realizations and the corresponding standard deviations of the means. We plot these values in Fig. 3.

For all hipster delay times $\tau$, the steady-state fraction $\rho_{B \text {,tot }}(t \rightarrow \infty)$ of nodes that are adopters of product $B$ increases rapidly for small $p_{\text {Hip. }}$. For $\tau=1$ [see Fig. 3(a)], the hipsters have access to information without any delays, and their behavior leads to a balancing of the adoptions of products $A$ and $B$. If a sufficiently large fraction of the nodes are hipsters, the mean final fraction of nodes that are adopters of one product is almost indistinguishable from the other. This occurs for $p_{\text {Hip }} \gtrsim 0.09$.

In all examined cases, $\rho_{B \text {,tot }}(t \rightarrow \infty)$ approximately equals $\rho_{A \text {,tot }}(t \rightarrow \infty)$ (i.e., the steady-state fraction of nodes 

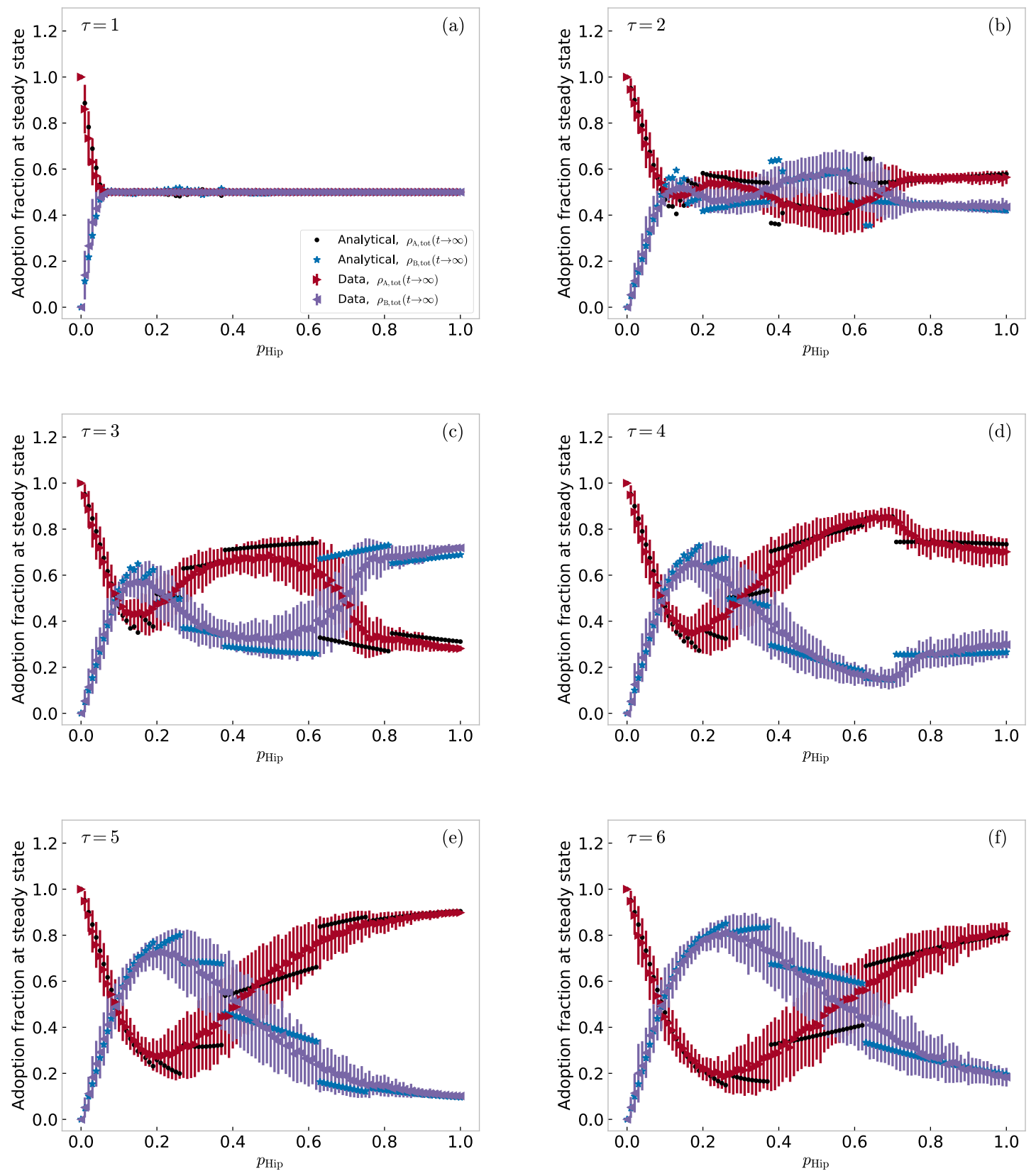

FIG. 3. Distribution of products at steady state for 10000-node 5-regular configuration-model networks. The different panels give results of simulations of our hipster threshold model with different delay times $\tau$ for the hipster nodes. For each value of $\tau$, we consider hipster probabilities $p_{\text {Hip }} \in[0,1]$ in increments of 0.01 . For each $\left(p_{\text {Hip }}, \tau\right)$ parameter pair, we simulate the hipster threshold model on 200 different networks that we construct using a configuration model (in which we connect stubs uniformly at random). The nodes have a threshold of $\phi=0.19$ with probability $p_{0}=0.8$ and threshold of $\phi=0.8$ with probability $1-p_{0}=0.2$. For each simulation, we activate a single node, chosen uniformly at random, with product $A$ at time $t=0$. We stop each simulation when product adoptions are no longer occurring. We plot the mean fraction of nodes that adopt products $A$ and $B$ in the 200 realizations and the corresponding standard deviations of the means. [For each $\left(\tau, p_{\text {Hip }}\right)$ parameter pair, we independently construct 200 networks, and we also independently determine the initial condition for each network.] For all values of $\tau$, the fraction of nodes that are adopters of product $B$ at steady state increases rapidly with $p_{\text {Hip }}$ for small $p_{\text {Hip }}$, reaching 0.5 at $p_{\mathrm{Hip}} \approx 0.09$. For $\tau=1$, which we show in panel (a), hipsters have information about the product distribution in the network without any delay, and the steady-state fractions of nodes that adopt products $A$ and $B$ are almost indistinguishable for $p_{\text {Hip }} \gtrsim 0.09$. For larger values of $\tau$, which we show in panels (b)-(f), the steady-state fraction of nodes that adopt each product varies for $p_{\text {Hip }} \gtrsim 0.09$. The steady-state fraction of nodes that adopt product $B$ is larger than that for product $A$ for an interval of $p_{\text {Hip }}$ values whose left end is at about $p_{\text {Hip }} \approx 0.09$.

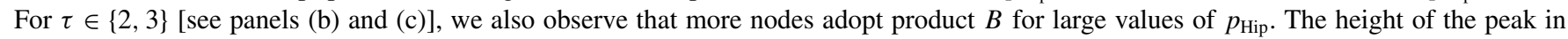
the fraction of product- $B$ adopters above $p_{\text {Hip }} \approx 0.09$ increases with $\tau$, reaching a value above 0.80 for $\tau=6$ [see panel (f)]. We also plot our analytically estimated product-adoption fractions from Eq. (1). Our analytical approximation matches the behavior well for small values of $p_{\text {Hip }}$ and large values of $p_{\text {Hip. }}$. Between these extremes, however, our approximation has jumps in the steady-state adoption fractions of products; these discontinuities do not arise in our numerical computations. 
that are adopters of product $A$ ) for $p_{\text {Hip }} \approx 0.09$. For $\tau \geqslant 2$ [see Figs. 3(b)-3(f)] and $p_{\text {Hip }} \gtrsim 0.09$, there exists an interval of $p_{\text {Hip }}$ values in which $\rho_{B \text {,tot }}(t \rightarrow \infty)>\rho_{A \text {,tot }}(t \rightarrow \infty)$. This interval is larger for larger values of $\tau$, and the peak of $\rho_{B \text {,tot }}(t \rightarrow \infty)$ in this interval grows with $\tau$, taking a value above 0.8 for $\tau=6$ [see Fig. 3(f)]. In other words, the fraction of hipsters must be larger than about 0.09 for product $B$ to be adopted by a larger fraction of the population than product $A$ at steady state.

For $\tau=2$ [see Fig. 3(b)], we observe another (and wider) $p_{\text {Hip }}$ interval (specifically, at about $[0.35,0.69]$ ) in which product $B$ beats product $A$. For $p_{\text {Hip }} \gtrsim 0.69$, product $A$ dominates. Hence, for $\tau=2$, product $B$ dominates in two $p_{\text {Hip }}$ intervals, and product $A$ dominates in three $p_{\text {Hip }}$ intervals. However, $\tau=3$ [see Fig. 3(c)] results in two intervals of dominance for each product. Product $B$ is the more-popular product at steady state in hipster-probability intervals starting at $p_{\text {Hip }} \approx 0.09$ and $p_{\text {Hip }} \approx 0.69$. Our simulations with $\tau \geqslant 4$ result in a single $p_{\text {Hip }}$ interval in which product $B$ is more popular than product $A$ at steady state. From the standard deviations, we see that different realizations with the same parameter values can yield rather different results.

Our analytical approximation and numerical computations match well for small and large $p_{\text {Hip }}$. However, our approximation includes jumps in the fraction of adopters for each of the products, and we do not observe such discontinuities in our simulations. The mean fraction of nodes that adopt a product in the discarded realizations is 0.0001 , which is much less than the threshold of 0.10 .

\section{B. 3-regular configuration-model networks}

We now examine our hipster threshold model on 3-regular configuration-model networks. Suppose that a probability $p_{0}=0.8$ of the nodes have a threshold of $\phi=0.3$ and that the remaining probability $1-p_{0}=0.2$ of the nodes have a threshold of $\phi=0.65$. We perform simulations as in the 5-regular configuration-model networks (see Sec. V A) and show our results in Fig. 4.

Our results on 3-regular configuration-model networks differ from those on 5-regular configuration-model networks in several ways. One interesting result is that the fractions that adopt products $A$ and $B$ are very similar for $\tau=2$ [see Fig. 4(b)] and $p_{\text {Hip }} \in[0.06,0.93]$. Additionally, for all examined $\tau \geqslant 3$ [see Figs. 4(c)-4(f)], the $\rho_{B \text {,tot }}(t \rightarrow \infty)$ curve on 3 -regular networks has one more maximum as a function of the hipster probability than the corresponding curve on the 5-regular configuration-model networks.

On 3-regular configuration-model networks with $\tau \geqslant 2$, we observe that $\rho_{B \text {,tot }}(t \rightarrow \infty)$ first becomes larger than $\rho_{A \text {, tot }}(t \rightarrow \infty)$ at about $p_{\text {Hip }} \approx 0.06$, which is lower than the hipster probability that we observed for the analogous result for 5-regular configuration-model networks. As we show in Fig. 5, this transition sometimes changes with seed size, depending on the value of the delay $\tau$. For $\tau=2$, the transition occurs at the same probability when we seed more nodes with product $A$; however, for the larger delay value $\tau=6$, the transition moves towards larger probabilities for progressively larger sets of seed nodes who adopt product $A$. When the seed size is 1 , we observe in Fig. 4 that the height of the first peak is lower when we simulate our model on 3-regular configuration-model networks than was the case for 5-regular configuration-model networks. For large $p_{\text {Hip }}$, the more-popular product at steady state is the same for $\tau=\{3,4\}$ [see Figs. 4(c) and 4(d)] as it is for the same delay times on the 5-regular configuration-model networks, while it is opposite to that on the 5-regular configuration-model networks for $\tau=\{5,6\}$ [see Figs. 4(e) and 4(f)]. For many parameter pairs, the standard deviations of the outcomes are large, indicating that realizations with identical parameters can yield very different outcomes.

Our analytical approximation and numerical simulations match well for $p_{\text {Hip }} \lesssim 0.05$. However, for values of $p_{\text {Hip }}$ that are larger than about 0.05 , our analytical approximation again has jumps that we do not observe in computations. Our analytical approximation also does not match the fraction of nodes that adopt each product for $p_{\text {Hip }}=1$ as well as it did on 5-regular configuration-model networks. This may be because 3-regular configuration-model networks have a higher edge density than 5-regular configuration-model networks, so the former depart rather significantly from satisfying a local-tree hypothesis (on which our analytical approximation relies). The mean fraction of nodes that adopt a product in the discarded realizations is 0.0002 , which is much less than the threshold of 0.10 .

\section{Erdôs-Rényi networks}

We now examine our hipster threshold model on ErdősRényi (ER) networks. Specifically, we examine $G(N, p)$ graphs, in which one specifies the total number $N$ of nodes, and each pair of nodes is linked independently with probability $p$. We choose the expected mean degree of the networks to be $z=5$ (so the probability of an edge between any two nodes is $p=z / N$ ) to match the mean degree of the 5-regular configuration-model networks that we examined in Sec. V A.

We assign the same threshold $\phi_{i}=\phi^{*}=0.2$ to each node. With this threshold, all nodes with degree $k \leqslant 5$ are vulnerable. We again consider $p_{\text {Hip }} \in[0,1]$ (in increments of 0.01 ) and $\tau \in\{1,2,3,4,5,6\}$. For each parameter pair ( $\left.\tau, p_{\text {Hip }}\right)$, we simulate the dynamics on 200 different networks, stop the simulations after reaching a steady state, track the final fractions of nodes that are adopters of each of the products, and calculate the corresponding mean and standard deviation of the mean from these data. As in prior simulations, we use a different set of 200 networks for each parameter value. We plot our results in Fig. 6.

As with our simulations on 5-regular and 3-regular configuration-model networks, the absence of time delay (i.e., $\tau=1$ ) in the information possessed by hipsters results in $\rho_{B \text {,tot }}(t \rightarrow \infty)$ being almost indistinguishable from $\rho_{A, \text { tot }}(t \rightarrow \infty)$ [see Fig. 6(a)]. For all examined values of $\tau$, we observe that $\rho_{B \text {,tot }}(t \rightarrow \infty)$ again increases rapidly for small values of $p_{\text {Hip. }}$. For $p_{\text {Hip }} \approx 0.07$, we observe that $\rho_{A \text {, tot }}(t \rightarrow \infty)$ and $\rho_{B \text {,tot }}(t \rightarrow \infty)$ have similar steady-state fractions, although one can also observe rather interesting dynamics. For large values of $p_{\text {Hip }}$, the same product becomes the more-popular one at steady state as with the 3-regular configuration-model networks for delay times $\tau=\{4,5,6\}$ [see Figs. 6(d)-6(f)], but product $A$ is the more-popular one 

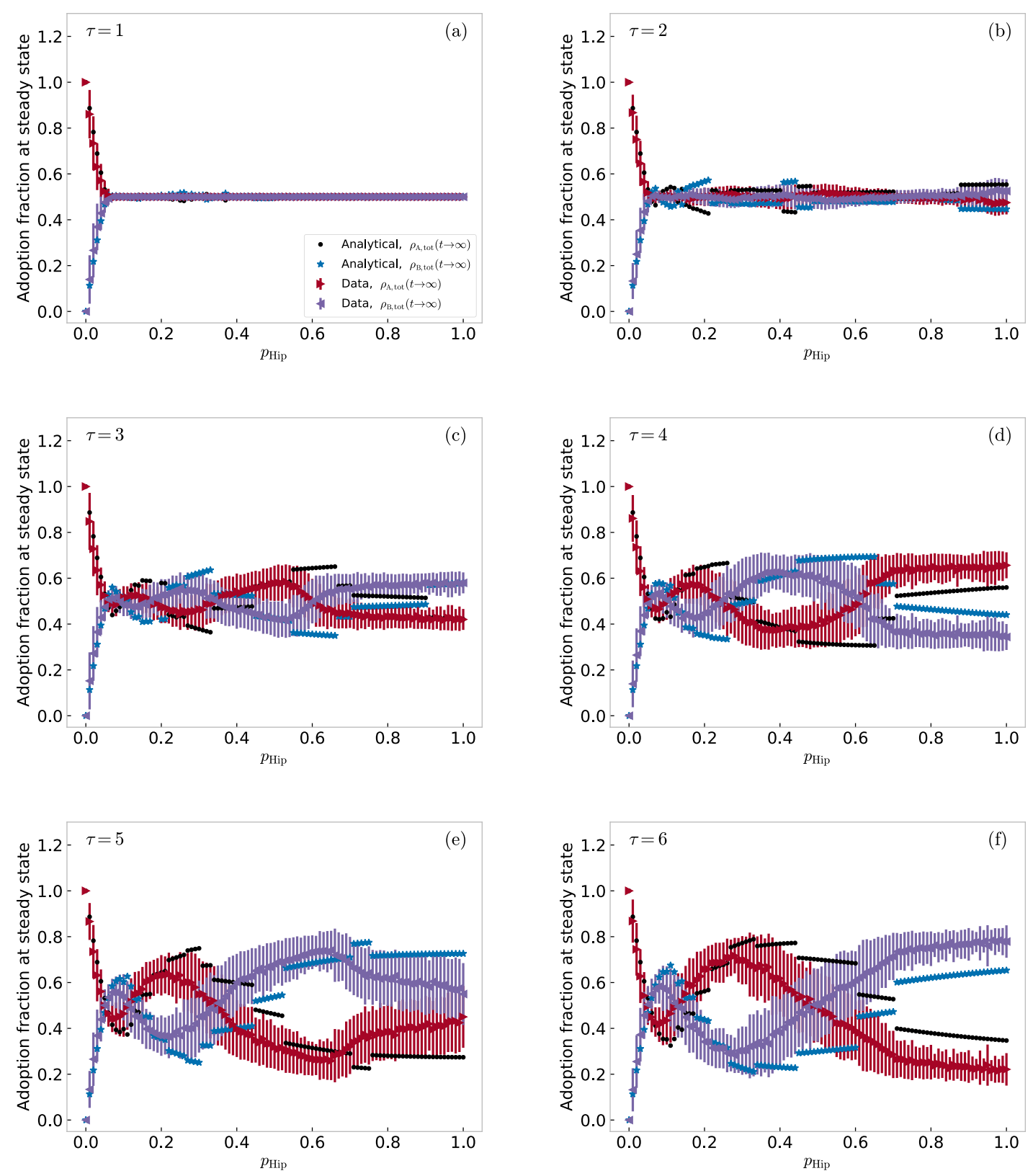

FIG. 4. Distribution of products at steady state for 10000-node 3-regular configuration-model networks. The different panels give results of simulations of our hipster threshold model with different delay times $\tau$ for the hipster nodes. For each value of $\tau$, we consider hipster probabilities $p_{\text {Hip }} \in[0,1]$ in increments of 0.01 . For each $\left(p_{\text {Hip }}, \tau\right)$ parameter pair, we simulate the model on 200 different networks that we construct using a configuration model (in which we connect stubs uniformly at random). The nodes have a threshold of $\phi=0.3$ with probability $p_{0}=0.8$ and threshold of $\phi=0.65$ with probability $1-p_{0}=0.2$. For each simulation, we activate a single node, chosen uniformly at random, with product $A$ at time $t=0$. We stop each simulation when product adoptions are no longer occurring. We plot the mean steady-state fraction of nodes that adopt products $A$ and $B$ in the 200 realizations and the corresponding standard deviations of the means. [For each $\left(\tau, p_{\text {Hip }}\right.$ ) parameter pair, we independently construct 200 networks, and we also independently determine the initial condition for each network.] For all values of $\tau$, the steady-state fraction of nodes that adopt product $B$ increases rapidly with $p_{\text {Hip }}$ for small $p_{\text {Hip }}$, reaching 0.5 at $p_{\text {Hip }} \approx 0.06$. For $\tau=1$, which we show in panel (a), hipsters have information about the product distribution in the network without any delay, and the steady-state fractions of nodes that adopt products $A$ and $B$ are almost indistinguishable for $p_{\text {Hip }} \gtrsim 0.06$. For $\tau=2$, which we show in (b), the fractions of nodes that adopt the two products are similar (though one can see some interesting dynamics) until $p_{\text {Hip }} \approx 0.93$, above which product $B$ is the more-popular product. For larger values of $\tau$ [see panels (c)-(f)], the fraction of nodes that adopt each product varies for $p_{\text {Hip }} \gtrsim 0.05$. The height of the peak, which occurs at $p_{\text {Hip }} \approx 0.08$, of the node fraction that adopts product $B$ increases with $\tau$, reaching a value of over 0.6 for $\tau=6$ [see panel (f)]. For all time delays $\tau \geqslant 2$ [see panels (b)-(f)], the maximum steady-state fraction that adopts product $B$ does not take place at $p_{\text {Hip }}$ values near 0.08 ; instead, it occurs for much larger values of $p_{\text {Hip }}$. We also plot our analytically estimated fractions of product adoption from Eq. (1). Our approximation matches well with our computations for small values of $p_{\text {Hip }}$. For $p_{\text {Hip }} \gtrsim 0.06$, however, our approximation does not do well. Our analytical solution includes jumps in the steady-state adoption fractions of the products, but these do not arise in our numerical simulations. 

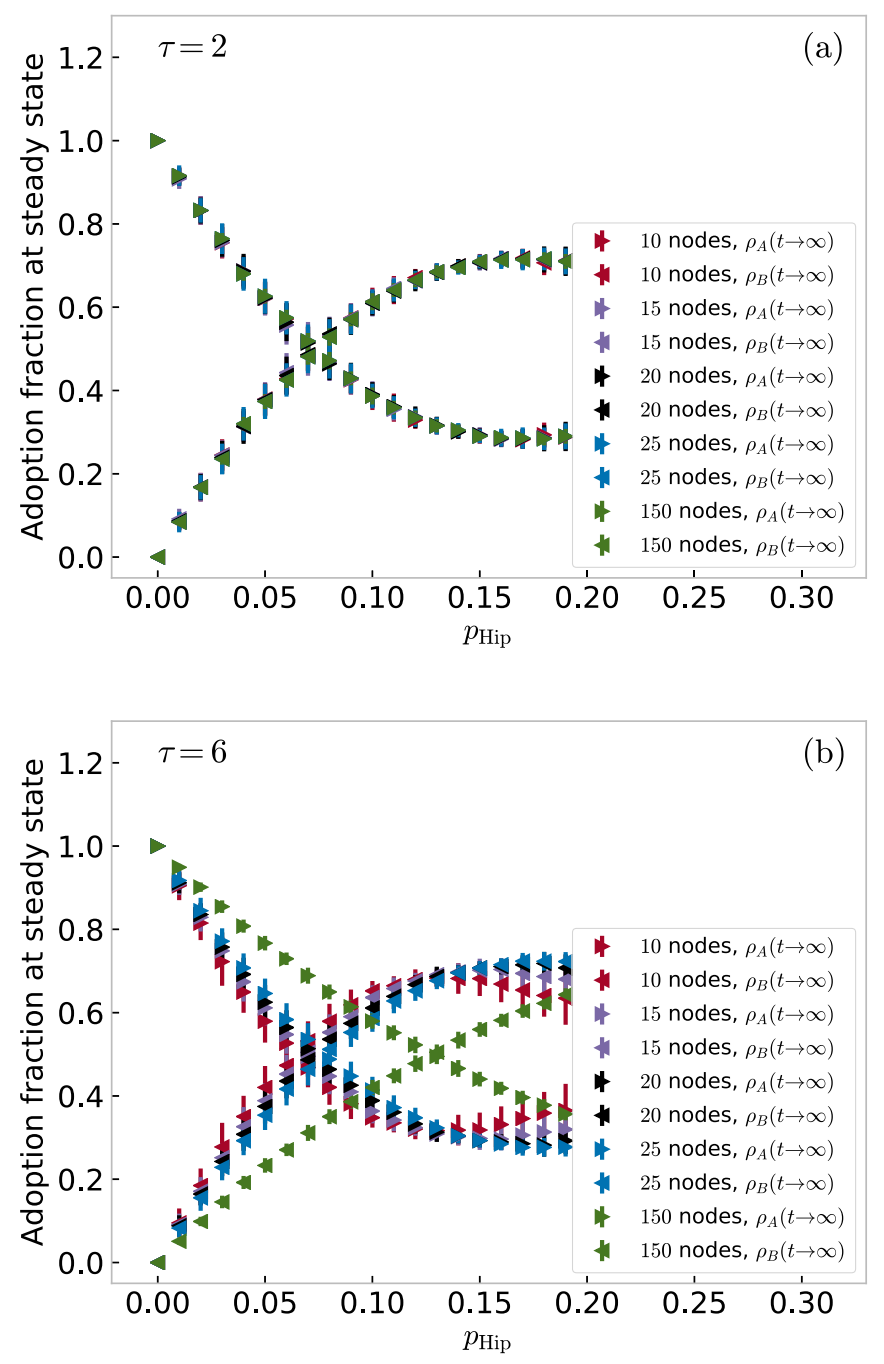

FIG. 5. Distribution of products at steady state for 10000 -node 3-regular configuration-model networks with different seed sizes, where all seed nodes adopt product $A$. We show the distribution of products for delays of (a) $\tau=2$ and (b) $\tau=6$. With $\tau=2$, the adoption fraction is indistinguishable for the different seed sizes. For $\tau=6$, our results vary for different seed sizes, but the qualitative behavior is consistent across all cases: the steady-state fraction of nodes that adopt product $B$ increases rapidly with $p_{\text {Hip }}$, and equal fractions adopt products $A$ and $B$ at a value of $p_{\text {Hip }}$ that increases slowly with seed size. For seed sets with 10 to 25 nodes, equal fractions of nodes adopt the two products at $p_{\text {Hip }} \approx 0.07$. For a seed set with 150 nodes, equal fractions adopt the two products at $p_{\text {Hip }} \approx 0.13$.

for $\tau=3$ [see Fig. 6(c)]. We generally observe large standard deviations of the outcomes of realizations with given parameter values. The mean fraction of nodes that adopt a product in the discarded realizations is 0.0072 . This is larger than what we observed for 3-regular and 5-regular configuration-model networks, but it is still much smaller than the threshold of 0.10 .

Our analytical approximation and numerical simulations once again match well for small values of $p_{\text {Hip }}$ (specifically, for $p_{\text {Hip }} \lesssim 0.07$ ). For larger values of $p_{\text {Hip }}$, our analytical approximation has jumps in the fraction that adopt each product; we again do not observe this phenomenon in our simulations. One possibility, which we suggested in our discussion of 3-regular configuration-model networks in Sec. VB, is whether our analytical approximation is running into problems because we are considering networks that are not locally treelike (although similar approximations are known to be effective for many networks that are not locally treelike [71]). Additionally, note that the mean local clustering coefficient for our ER networks with $z=5$ is $0.00058 \pm 0.00018$, so our networks have very few 3 -cycles. If we ignore which product is adopted and pretend that the two products are the same, we recover the usual WTM model; the present paper uses an analytical approximation that is known to work in that situation [31]. Our own recent work has demonstrated that this type of analytical approximation is also effective for a WTM augmented with "synergistic" social influence from nodes other than nearest neighbors [27], so the incorporation of different types of nodes (rather than the lack of a locally treelike network structure) appears to be the likely cause of the breakdown of the approximation, especially given that our approximation becomes worse as we increase the hipster probability $p_{\text {Hip }}$.

\section{The NORTHWESTERN25 Facebook network}

In Sec. V B, we showed simulations of our hipster threshold model model on the NORTHWESTERN25 network from the FACEBOOK100 data set for two choices of the $\left(p_{\text {Hip }}, \tau\right)$ parameter pair. We now examine our model on the NORTHWESTERN25 network more systematically by considering more initial conditions and a wider variety of parameter values. Suppose that each node has a threshold of $\phi^{*}=1 / 33$. In each of our simulations, we use a single node, chosen uniformly at random, as a seed at $t=0$ and consider $\tau \in\{1,2,3,4,5,6\}$ and $p_{\text {Hip }} \in[0,1.0]$ (in increments of 0.01 ).

In Fig. 7, we show the mean fraction of nodes that are adopters of products $A$ and $B$ at steady state. For each choice of parameters, we choose a set of 200 initial conditions, and we calculate means over these simulations. The most striking difference between these plots compared to those for our model on synthetic networks in previous sections is that now it takes more hipsters to obtain equal steady-state fractions of adopters of the two products. In the NORTHWESTERN25 network, the fractions that adopt the two products become equal when roughly one fifth of the nodes are hipsters. We also observe that the height of the first peak of $\rho_{B \text {, tot }}(t \rightarrow \infty)$ increases with $\tau$, as was also the case in the synthetic networks that we examined, and the standard deviations are once again large for most parameter pairs. The mean fraction of nodes that adopt a product in the discarded realizations is 0.0001 , which again is much less than the threshold of 0.10 .

\section{MAJOR IMPACT OF A FEW INDIVIDUALS: APPROXIMATION ON $\boldsymbol{k}$-REGULAR TREES}

In Sec. V, we observed that even just a few hipster nodes can cause product $B$ to become the more-popular product at steady state, but we have not yet explored how this phenomenon can occur. In this section, we argue why even just a few antiestablishment nodes can have a major impact on 

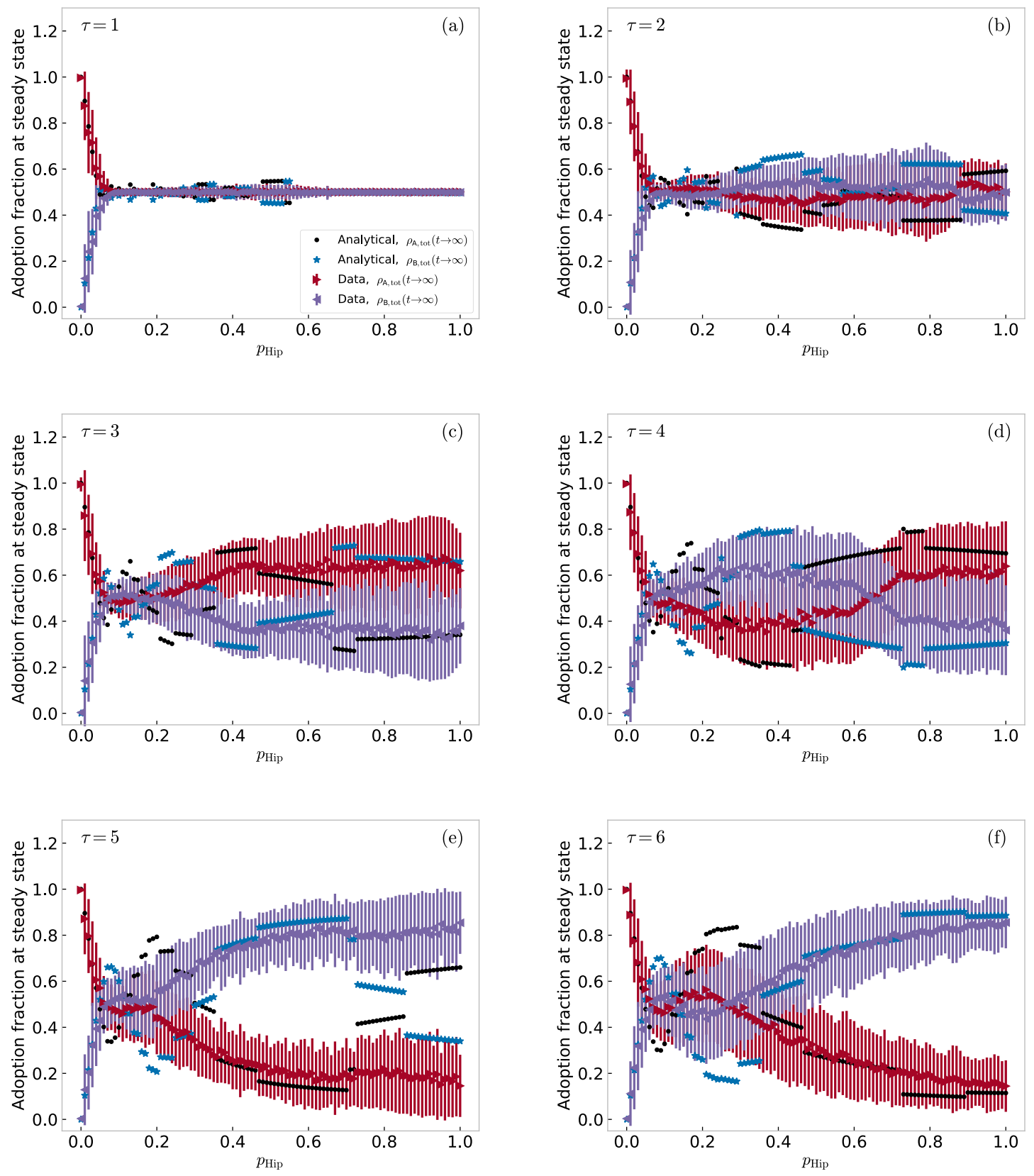

FIG. 6. Distribution of products at steady state for 10000 -node Erdôs-Rényi networks with an expected mean degree of $z=5$. The different panels give results of simulations of our hipster threshold model with different delay times $\tau$ for the hipster nodes. For each value of $\tau$, we consider hipster probabilities $p_{\text {Hip }} \in[0,1]$ in increments of 0.01 . For each $\left(\tau, p_{\text {Hip }}\right.$ ) parameter pair, we simulate the model on 200 different networks and initial conditions. Each node has a threshold of $\phi=0.2$. For each simulation, we activate a single node, chosen uniformly at random, with product $A$ at time $t=0$. We stop each simulation when product adoptions are no longer occurring. We plot the mean steady-state fraction of nodes that adopt products $A$ and $B$ in the 200 realizations and the corresponding standard deviations of the means. [For each $\left(\tau, p_{\text {Hip }}\right)$ parameter pair, we independently construct 200 networks, and we also independently determine the initial condition for each network.] For all values of $\tau$, the fraction of nodes that adopt product $B$ increases rapidly with $p_{\text {Hip }}$ for small $p_{\text {Hip }}$, reaching 0.5 at $p_{\text {Hip }} \approx 0.07$. For $\tau=1$, which we show in panel (a), hipsters have information about the product distribution in the network without any delay, and the steady-state fractions of nodes that adopt products $A$ and $B$ are almost indistinguishable for $p_{\text {Hip }} \gtrsim 0.07$. For larger values of $\tau$ [see panels (b)-(f)], the steady-state fraction of nodes that adopt each product varies for $p_{\text {Hip }} \gtrsim 0.07$. For all $\tau \geqslant 3$ [see panels (c)-(f)] the fraction of nodes that adopt product $B$ is largest for a small interval of $p_{\text {Hip }}$ around $p_{\text {Hip }} \approx 0.10$. For $\tau \geqslant 4$ [see panels (d)-(f)], we observe an additional, large- $p_{\text {Hip }}$ interval in which a majority of the nodes adopt product $B$. We also plot our analytically estimated fractions of product adoption from Eq. (1). Our analytical curves and numerical simulations match well for small values of $p_{\text {Hip }}$. For larger hipster probabilities, however, our analytical approximation is not accurate. For $\tau=5$ [see panel (e)], it predicts incorrectly that product $A$ is the more-popular product at steady state for large values of $p_{\text {Hip }}$. Our analytical results also include jumps in the steady-state adoption fractions of products that are not present in our numerical simulations. 

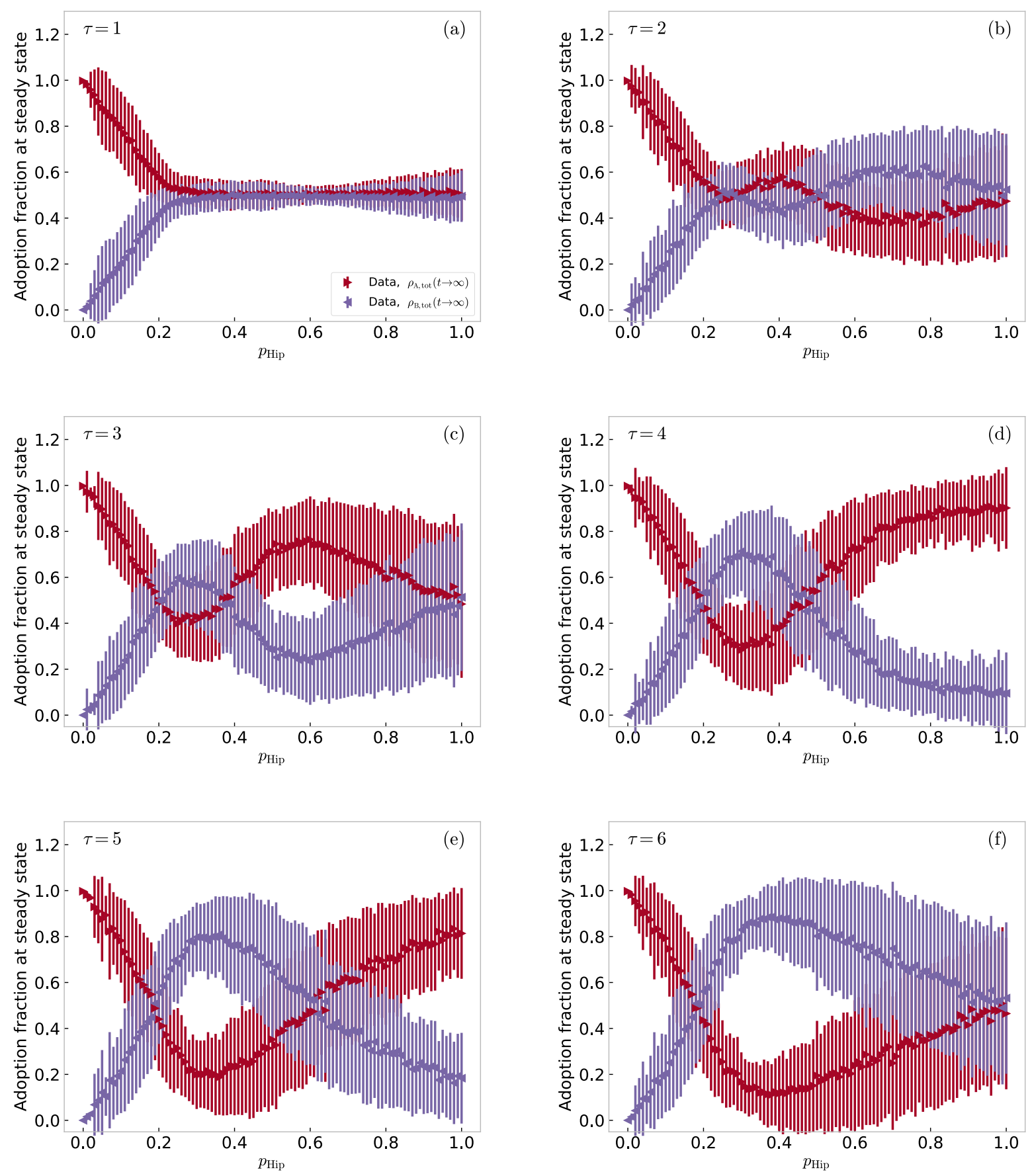

FIG. 7. Distribution of products at steady state for the NORTHWESTERN 25 network from the FACEBOOK 100 data set. The different panels give results of simulations of our hipster threshold model with different delay times $\tau$ for the hipster nodes. For each value of $\tau$, we consider hipster probabilities $p_{\text {Hip }} \in[0,1]$ in increments of 0.01 . For each $\left(\tau, p_{\text {Hip }}\right)$ parameter pair, we simulate the hipster threshold model on the NORTHWESTERN 25 network with 200 choices for the seed node, chosen uniformly at random, which adopts product $A$ at $t=0$. We use a different set of 200 nodes for different parameter values. Each node has a threshold of $\phi=1 / 33$. We plot the mean fractions of nodes that are adopters of products $A$ and $B$ at steady state in the 200 realizations and the corresponding standard deviations of the means. For all values of $\tau$, the steady-state fraction of nodes that adopt product $B$ increases rapidly with $p_{\text {Hip }}$ for small $p_{\text {Hip }}$, reaching 0.5 at $p_{\text {Hip }} \approx 0.2$ for $\tau \geqslant 3$ [see panels (c)-(f)] and for larger values of $p_{\text {Hip }}$ for $\tau \leqslant 2$ [see panels (a) and (b)]. For $\tau=1$, which we show in panel (a), hipsters have information about the product distribution in the network without any delay, and the steady-state fractions of nodes that adopt products $A$ and $B$ are very similar for $p_{\text {Hip }} \gtrsim 0.3$. For larger values of $\tau$ [see panels (b)-(f)], the fraction of nodes that adopt each product varies nonmonotonically for $p_{\text {Hip }} \gtrsim 0.2$. For $\tau \geqslant 3$ [see panels (c)-(f)], the fraction of nodes that adopt product $B$ is largest for a small interval of $p_{\text {Hip }}$ around $p_{\text {Hip }} \approx 0.3$. This is the single peak in the adoption of product $B$ in the mean over these simulations. For $\tau=2$ [see panel (b)], product $B$ is the more-popular product for large values of $p_{\text {Hip. }}$. For $\tau \geqslant 2$ [see panels (b)-(f)], product $B$ is the more-popular product for a $p_{\text {Hip }}$ interval starting at $p_{\text {Hip }} \approx 0.20$. The length of this interval increases with $\tau$, and both the hipster probability that produces the peak fraction in this interval and (especially) the value of the peak fraction increase with $\tau$. For $\tau=6$ [see panel (f)], the maximum fraction of nodes that adopt product $B$ is about 0.90 . 
(a)
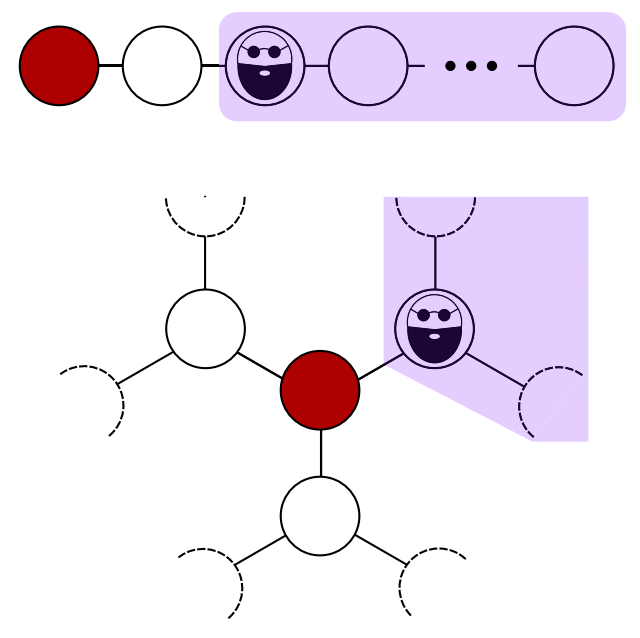

FIG. 8. (a) A line graph in which the leftmost node is the only seed. (It adopts product $A$.) If all nodes are vulnerable and exactly one node is a hipster, all nodes to the hipster's right eventually adopt product $B$. (b) A 3-regular tree in which the central node is the only seed. (It adopts product $A$.) If the tree has a single hipster, all nodes that are descendants of the hipster eventually adopt product $B$.

steady-state adoptions in our model. From studying this mechanism, we expect that some similar qualitative phenomena occur in many other models, including ones with stochastic update rules.

To understand why even a few hipster nodes can dramatically increase the number of product- $B$ adopters at steady state, we first consider a line of $N$ nodes, which we number from one end to the other with the labels $0,1,2, \ldots, N-1$. Each node is adjacent to its immediate neighbors, with 2 neighbors each, except for nodes 0 and $N-1$ (which each have degree 1). For the sake of the argument, we assume that all nodes are vulnerable and that there is no delay in information (so that $\tau=1$ ). We also suppose that node 0 is the only seed, so it has adopted product $A$ at time $t=0$ [see Fig. 8(a)]. If there are no hipsters in the line, all nodes in this scenario eventually adopt product $A$. If, by contrast, a single node $i$ is a hipster, then all nodes $j \geqslant i$ eventually adopt product $B$. Therefore, if each node has the same independent probability of being a hipster, the expected steady-state fraction of product- $B$ adopters approaches $1 / 2$ as $N \rightarrow \infty$. In this case, the presence of a single hipster node increases the expected steady-state fraction of nodes that adopt product $B$ from 0 nodes to half of the nodes. The main idea is that early adopters can influence later adopters in a way that depends on the adoption paths that are available [39]. Moreover, although the expected steady-state fraction of product- $B$ adopters is $1 / 2$, a single simulation of the model is equally likely to result in any number of product- $B$ adopters, because each node is equally likely to be the hipster. This may be a reason why we observe large standard deviations in different realizations of our model on the various types of networks. For more complicated network topologies, although it is no longer true in general that different steady-state fractions of product$B$ adopters are equally probable, the steady-state adoption fraction in a given simulation depends significantly on where hipsters are located in a network.

With this simple example in mind, we now turn to a more difficult example: a $k$-regular tree of vulnerable nodes in which the central node (which we label as node 0 ) is the only seed [see Fig. 8(b)]. As usual, the seed has adopted product $A$. As in the above example on a line graph, if a certain node is a hipster, it will force the nodes that follow it in an adoption path to adopt product $B$, rather than product $A$. We can divide the tree into hierarchical "levels." The central node is 0 , and it is adjacent to $k$ nodes in level 1. Each node in level 1 is adjacent to $k-1$ nodes in level 2, each node in level 2 is adjacent to $k-1$ nodes in level 3 , and so on. Hence, level $l \geqslant 1$ includes $n_{l}=k(k-1)^{l-1}$ nodes, and all nodes except those in the last level (which have degree 1) have degree $k$. Such a $k$-regular tree with $L$ levels has $N=1+\sum_{l=1}^{L} k(k-$ $1)^{l-1}$ nodes. In the limit of infinitely many levels, a $k$-regular tree is a Bethe lattice.

Suppose that there is a single hipster in the network. By construction, we can view any hipster as the root in a rooted tree. We can then make the following approximation. If all nodes have an equal, independent probability of being a hipster, the probability for there to be a hipster in level $l$ is equal to the fraction of nodes $\left(n_{l} / N\right)$ that are in that level. If a hipster is present in level $l$, all nodes in an adoption path after that hipster (i.e., all of its descendants) eventually adopt product $B$. Because level $l$ has $n_{l}$ nodes, a single hipster in level $n_{l}$ causes $1 / n_{l}$ of the nodes in later levels $\left(l^{\prime} \geqslant l+1\right)$ to adopt product $B$. Therefore, one can approximate the expected steady-state fraction of product- $B$ adopters as

$$
\begin{aligned}
\bar{\rho}_{B}\left(n_{\text {Hip }}=1\right) & \approx \sum_{s=1}^{L} \frac{n_{s}}{N} \frac{1}{n_{s}}\left[\frac{1}{N} \sum_{l=s}^{L} k(k-1)^{l-1}\right] \\
& =\frac{1}{N^{2}} \sum_{s=1}^{L} \sum_{l=s}^{L} k(k-1)^{l-1} .
\end{aligned}
$$

For a spreading process on a network, one can construct a dissemination tree, which describes how a contagion spreads through the network [39]. For a $k$-regular tree with only vulnerable nodes, the dissemination tree is the same $k$-regular tree, except that all edges are directed from the center towards the periphery. The above analysis indicates that the fraction of nodes that a single hipster can cause to adopt product $B$ is related to the properties of a dissemination tree. For dissemination trees with a progressively larger number of mean descendants per node, we expect a progressively larger fraction of nodes in an associated network to adopt product $B$ when a single hipster is present in the network. Equation (5) illustrates that, for a given network, increasing the number of hierarchical levels in a dissemination tree tends to result in a larger number of product- $B$ adopters from a single hipster.

To obtain a naive estimate of the fraction of product- $B$ adopters as a function of $n_{\text {Hip }}$ when $n_{\text {Hip }} \ll N$, we multiply Eq. (5) by $n_{\text {Hip }} / N$, thereby assuming that adding a second hipster to the network leads to as many product- $B$ adopters as the number that resulted from the original hipster [76]. However, the second hipster may be a descendant of the existing hipster, such that it does not lead to any additional product- $B$ 
adoptions. To account for this, we develop a recursive formula that takes this possibility into account.

Imagine adding hipsters to a network one at a time (allowing the possibility of choosing the same node multiple times when attempting to add hipsters). We seek to approximate the expected fraction of product- $B$ adopters at steady state in a network with $n_{\text {Hip }}$ hipsters as a function of the expected fraction of product- $B$ adopters at steady state in a network with $n_{\text {Hip }}-1$ hipsters. Let $P_{\text {desc }}$ denote the probability that the additional hipster is a descendant of another hipster in the network. Adding a hipster has two possible outcomes: (1) the hipster is a descendant of another hipster, such that it does not yield additional product- $B$ adopters; or (2) the hipster is not a descendant of another hipster, so on average it yields another $\bar{\rho}_{B}\left(n_{\text {Hip }}=1\right)$ fraction of product- $B$ adopters at steady state. We summarize this reasoning in the formula

$$
\begin{aligned}
\bar{\rho}_{B}\left(n_{\text {Hip }}\right) \approx & \bar{\rho}_{B}\left(n_{\text {Hip }}-1\right) P_{\text {desc }} \\
& +\left[\bar{\rho}_{B}\left(n_{\text {Hip }}-1\right)+\bar{\rho}_{B}\left(n_{\text {Hip }}=1\right)\right]\left(1-P_{\text {desc }}\right) .
\end{aligned}
$$

In a $k$-regular tree with sufficiently few hipsters, all descendants of a hipster are product- $B$ adopters at steady state, so the probability that the $n$th hipster descends from one of the previous $n-1$ hipsters equals the expected fraction of nodes that are product- $B$ adopters at steady state in a network with $n_{\text {Hip }}=n-1$ hipsters. We thus insert $P_{\text {desc }}=\bar{\rho}_{B}\left(n_{\text {Hip }}-1\right)$ into Eq. (6) to obtain

$$
\bar{\rho}_{B}\left(n_{\text {Hip }}\right) \approx \bar{\rho}_{B}\left(n_{\text {Hip }}-1\right)+\left[1-\bar{\rho}_{B}\left(n_{\text {Hip }}-1\right)\right] \bar{\rho}_{B}\left(n_{\text {Hip }}=1\right) .
$$

In Fig. 9, we compare the analytical expression in Eq. (7) to computations using 3-regular and 5-regular trees. Our analytical approximation is a good match for our simulations when $n_{\text {Hip }} / N$ is small. For larger $n_{\text {Hip }} / N$, Eq. (7) overestimates the steady-state fraction of nodes that adopt product $B$. Hipsters need not always adopt product $B$; with more hipsters, it becomes increasingly likely that product $B$ is not always the less-popular product.

Our analysis has several interesting consequences. For example, it yields some understanding of how the delay $\tau$ affects the steady-state adoption fractions of each product. To illuminate the impact of $\tau$, it is helpful to consider the following situation. Suppose that, because of hipsters, product $B$ becomes more popular than product $A$ at some point during a simulation of our model. A delay of $\tau \geqslant 2$ postpones this time, at which hipsters start adopting product $A$ rather than product $B$, so we expect hipsters who adopt product $A$ to have fewer descendants than if $\tau=1$. This provides an argument for why the height of the peak of the fraction of product- $B$ adopters as a function of $p_{\text {Hip }}$ increases with the delay, and it sheds some light on the effects of the delay. If there is no delay (i.e., $\tau=1$ ) and there are many hipsters, then hipsters tend to balance the popularities of the two products, leading to roughly equal fractions of the two products at steady state (as we saw in our simulations on all networks in Sec. V).

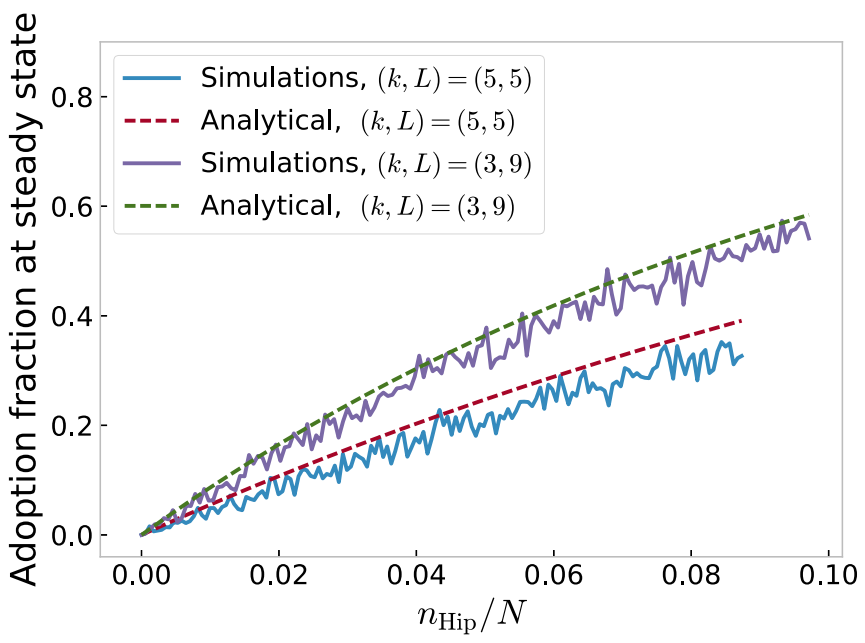

FIG. 9. Fraction of nodes in $k$-regular trees with $L$ levels that are adopters of product $B$ at steady state. We show results for 3-regular trees with 9 levels (and hence with $N=1534$ nodes in total) and for 5-regular trees with 5 levels (and hence with $N=1706$ nodes in total). We plot the recursive approximation from Eq. (7) and show our simulation results, averaged over 100 realizations, for the steady-state fraction of nodes that adopt product $B$ as a function of the fraction $n_{\text {Hip }} / N$ (with $n_{\text {Hip }} \in\{0,1, \ldots, 150\}$ ) of hipsters in the network. As expected, our approximation is good for $n_{\text {Hip }} \ll N$.

Our analysis also improves our understanding of how various changes to our hipster model can affect steady-state results. For example, suppose that we use a stochastic updating rule instead of a deterministic one. Although the above analysis does not rely on the deterministic nature of our updating rule, it does indicate that adoption order is important, and anything that changes the adoption order (such as using a stochastic update rule or updating node states asynchronously instead of synchronously) may change the outcome of simulations [77]. However, from our analysis, we do expect some features of our results to be robust even with different update rules and update orders. For example, for either stochastic update rules or asynchronous updating, we expect an increase in the number of steady-state product- $B$ adopters with increasing $p_{\text {Hip }}$ for small values of $p_{\text {Hip }}$, followed by a decrease (or stall) in the number of steady-state product- $B$ adopters when enough hipsters are present in a network (as some of them will now adopt product $A$ ). However, the rate at which the steadystate fraction of product- $B$ adopters increases with $p_{\text {Hip }}$ for small $p_{\text {Hip }}$ is likely to be influenced by stochastic update rules and asynchronous updating. For instance, suppose that we use the same rules for product selection but that we employ an asynchronous updating process in which, during each time step, we select a node uniformly at random to update; we repeat this selection process some number of times during the same time step; and we then advance time by one step. We then continue with this process in our simulations until no further spreading occurs. In this case, every node in a network can potentially adopt a product even in the first time step, and the process tends to spread at a different rate-it can be either faster or slower - than in synchronous updating. Because the hipsters are distributed uniformly at random 
and the rules governing product choice are the same as in our original model, changing the number of adopters during each time step can directly affect hipsters, as their product choice is time-dependent. (Other nodes are affected indirectly, as they can experience a different product distribution in their neighborhoods.) Consequently, a faster initial spreading would increase early product- $B$ adoption for a delay $\tau \geqslant 2$, as all hipsters are guaranteed to choose product $B$ for time steps $t \leqslant \tau$. To test this, we simulate the spreading of products on 5-regular configuration-model networks with $\tau=2$ and a single product- $A$ adopter as a seed. We use the asynchronous updating procedure that we just described above. Averaging our results over 100 realizations [which we determine as in Fig. 3(b)], we find that the steady-state product- $B$ adoption fraction increases faster as we increase $p_{\text {Hip }}$ for small values of $p_{\text {Hip }}$ than what was the case for synchronous updating [see Fig. 3(b)]. As we expected, we also find that the product- $B$ steady-state adoption fraction decreases as we increase $p_{\text {Hip }}$ for larger values of $p_{\text {Hip. }}$. More generally, different update mechanisms and update orders can yield different dissemination trees, which describe how a contagion spreads through a network [39]. This can, in turn, impact steady-state product popularities.

Another aspect that tends to alter a dissemination tree is changes in the threshold distribution of nodes in a network. For example, with a threshold distribution in which all nodes are vulnerable, a spreading process can reach a steady state very quickly, and there are then few hierarchical levels in the associated dissemination tree. By contrast, a threshold distribution for which a network starts with fewer vulnerable nodes may take longer to reach a steady state, and one thus expects more levels in an associated dissemination tree. From our analysis, we see that this in turn can increase the steady-state adoption fraction of product $B$ for small values of $p_{\text {Hip }}$. Performing simulations on 5-regular configuration model networks with $\tau=2$ [as in Fig. 3(b)] and 150 seed nodes with probabilities $p_{0}=1.00, p_{0}=0.90$, and $p_{0}=$ 0.80 supports this intuition. When we examine small values of $p_{\text {Hip }}$, the steady-state fraction of product- $B$ adopters increases slightly more slowly for larger values of $p_{0}$ as we increase $p_{\text {Hip }}$.

For some network families, we expect networks with different numbers of nodes to have different fractions of product$B$ adopters at steady state. To illustrate this point, we again consider line networks and $k$-regular trees. For a line network with a single hipster and a seed node that adopts product $A$ at one end, the expected steady-state fraction of product- $B$ adopters is roughly $1 / 2$ for a line with any number of nodes. However, adding another level to a $k$-regular tree with a single seed node that adopts product $A$ affects the expected steadystate fraction of product- $B$ adopters. For example, a 3-regular tree with 3 levels (and hence with 10 nodes in total) has an expected steady-state fraction $\bar{\rho}_{B}=15 / 100$ of product- $B$ adopters, whereas a 3-regular tree with 4 levels (and hence with 22 nodes in total) has $\bar{\rho}_{B}=1 / 10$. This difference occurs because adding another level to the 3-regular tree increases the fraction of nodes that are leaves. Therefore, the randomly distributed hipsters have fewer descendants on average in a dissemination tree, decreasing the expected fraction of product- $B$ adopters at steady state. In simulations on regular configuration-model networks with $10^{3}$ nodes (using several values of $\tau$ for 3-regular networks and $\tau=3$ for 5-regular ones) for small values of $p_{\text {Hip }}$, we observe the same fast increase in steady-state product- $B$ adopters as we increase $p_{\text {Hip }}$ that we observed previously for these networks with $10^{4}$ nodes (see, e.g., Fig. 4). However, for large values of $p_{\text {Hip }}$, the steady-state product- $B$ adopter fraction can differ substantially in simulations on networks with $10^{3}$ nodes and $10^{4}$ nodes. More generally, our analysis demonstrates that the number of nodes in a network can affect steady-state product distributions. Even taking seed-size scaling into consideration (see [70]), dissemination trees can still change, potentially affecting qualitative steady-state results.

Changing the way that nodes choose which product to adopt can also drastically influence simulation outcomes. For example, consider a modification of our model in which a hipster that becomes active at time step $t$ adopts the product that is less popular among its neighbors at time $t-\tau$. Furthermore, suppose that two competing products are spreading in a $k$-regular tree in which the central node is the only seed. As usual, the seed has adopted product $A$. When we constructed our approximation (7) for the steady-state distribution of products in the limit of few hipsters, we assumed that every hipster adopts product $B$. In the modified hipster model in our current discussion, this approximation may be very bad. Hipsters who descend from other hipsters may adopt product $A$. We thus expect the product- $B$ steady-state adoption fraction to increase more slowly with $p_{\text {Hip }}$ for small $p_{\text {Hip }}$ if a hipster adopts the product that is less popular among its neighbors, rather than the less-popular product among all active nodes in a network. Performing simulations of the modified hipster model on 5-regular configuration-model networks with $p_{0}=$ 0.80 vulnerable-node probability, $\tau=1$, and a single seed node [as in Fig. 3(a)] for $p_{\text {Hip }} \leqslant 0.14$, we find that a smaller (or equal, for $p_{\text {Hip }}=0$ ) steady-state fraction of nodes adopts product $B$ than in our observations for our focal hipster model.

\section{CONCLUSIONS}

It is important to study what makes information, opinions, diseases, memes, products, misinformation, alternative facts, and other things that originate in a small subpopulation spread to a large fraction of nodes in a network. Such scenarios can arise in the adoption of products and the spreading of memes, and they can also occur in antiestablishment behavior, which can significantly impact the geopolitical landscape.

We developed a threshold model to examine the impact of anticonformists (so-called "hipsters") on the spreading of two competing products (one of which, labeled $B$, is not adopted by any node at the beginning of our simulations). We examined our hipster threshold model on various types of networks, and we considered different probabilities of the hipster nodes and different amounts of time delay in the global information that the hipsters possess. In the absence of a time delay, we found that hipsters tend to balance the adoption of the two competing products. For all other delay values and all examined types of networks, we observed that the steady-state fraction of nodes that adopt product $B$ (i.e., the 
product that would not be adopted in the absence of hipsters) grows rapidly with the probability of hipsters. Surprisingly, for all of our networks, we needed only a small probability of hipsters to observe many situations in which product $B$ is comparably popular, or even more popular, than product $A$ at steady state. In our simulations on a variety of synthetic networks, we found that it is often sufficient for fewer than $10 \%$ of the nodes to be hipsters for product $B$ to become at least as widespread as product $A$ (the only product that has any adopters at time $t=0$ ). For the NORTHWESTERN25 Facebook network, roughly $20 \%$ of the nodes need be hipsters for product $B$ to be as widespread as product $A$ at steady state.

Using a line network and $k$-regular trees, we illustrated why the fraction of nodes that adopt product $B$ increases rapidly for small values of $p_{\text {Hip }}$. On these networks, we obtained good agreement between simulations and an approximation of the steady-state fraction of nodes that adopt product $B$ in the limit of few hipsters. From our analytical approximation in the few-hipster regime, we observed that the steady-state product- $B$ adoption fraction increases with the distance between the seed node and other nodes. This gives some insight into why there is a much slower increase in product- $B$ adopters for the spreading process on the Facebook network than in the examined synthetic networks, as the former has a smaller mean geodesic (i.e., shortest) path length than our synthetic networks. It also suggests that different realizations with identical parameter values may result in very different steady-state adoption fractions, given that we use random processes to choose hipsters and seed nodes. One consequence of such sensitivity to initial conditions is large standard deviations in the mean steady-state adoption fraction of each product, which is what we observed in most cases. The same mechanistic insight suggests that a larger delay $\tau$ results in more hipsters adopting product $B$ early in a simulation, and each of these early adopters influences the product choice of later adopters. We believe that postponing the time at which hipsters choose product $A$ instead of product $B$ is the main reason that a progressively larger delay $\tau$ results in a progressively larger peak of the expected product- $B$ adoption fraction as a function $p_{\text {Hip }}$. Finally, the mechanistic insight from our approximation in the few-hipster limit also helps illustrate that the properties-such as threshold distributions, the number of nodes in a network, and update rules - of an update rule or network that affect dissemination trees (which describe how a contagion spreads through a network) can affect observations at steady state, although some qualitative observations should be robust under such variations.

Our hipster threshold model exhibits a variety of fascinating dynamics on different types of networks. For example, when there is a delay in global information (i.e., $\tau \geqslant 2$ ) and the hipster probability $p_{\text {Hip }}$ is large, we observed nontrivial characteristics in the number of intervals of hipster probabilities for which a given product is more popular at steady state. The quality of the match between our pair approximation and numerical simulations also depends both on network structure and on the hipster probability. For example, our approximation was effective for small values of $p_{\text {Hip }}$, and it correctly produced a fast increase in product- $B$ adopters with increasing values of small $p_{\text {Hip }}$; it did reasonably well for large values of $p_{\text {Hip }}$ for 5-regular configuration-model networks (except for abrupt jumps that are not present in the simulations); it achieved mixed results for 3-regular configuration-model networks (although it yielded the correct result for the morepopular product at steady state for $p_{\text {Hip }} \approx 1$ in all but one instance); and it was ineffective for Erdős-Rényi networks (where it was incorrect about which product is more popular at steady state for $p_{\text {Hip }} \approx 1$ in roughly half of the cases).

When there is a delay (i.e., $\tau \geqslant 2$ ) in the global adoption information that is available to hipsters, we also found that the steady-state fraction of nodes that adopt a product varies nonmonotonically with the probability of hipsters. For some delay values, this steady-state fraction peaks for multiple, disparate values of the probability of hipsters; for other delay values, however, there is only a single peak. This behavior also depends on the network type on which spreading occurs. If there is no delay in the global adoption information that is available to hipsters (i.e., $\tau=1$ ), we found that the steadystate fraction of nodes that adopt product $B$ first increases rapidly with $p_{\text {Hip }}$ and then stabilizes, such that approximately half of the nodes adopt each product.

In summary, in our hipster model, even when only one of two products is adopted when spreading begins, small probabilities of antiestablishment nodes can lead to a competitor product being adopted by a majority in a population. Our simple model and numerical experiments may help shed light on the road to success for antiestablishment choices in elections and competition between products, as such success (and qualitative differences in final outcomes between competing products, political candidates, and so on) can arise rather generically from a small number of antiestablishment individuals and ordinary processes of social influence on normal individuals. In our model, the hipsters always choose to adopt the product that is less popular at time step $t-\tau$. If all hipsters regard product $A$ as the established choice at all time steps-regardless of the actual distribution of adopted products - the steady-state adoption fractions of product $B$ become even larger, and the antiestablishment choice (which is product $B$, in our example) becomes even more successful than what we observed in our simulations. This more extreme situation may be relevant in elections in which the conception of who is part of the establishment may not change during weeks of campaigning and polls that forecast which candidate will win and take office.

In future work, it would be interesting to study our hipster model in more detail, including investigating whether the fraction of hipsters is connected to any notion of criticality, and to extend the model in various ways. Generalizations of our model may be helpful for studying the impact of antiestablishment hubs, such as alt-right broadcasting services or alt-right Twitter accounts with many followers. Understanding what makes a large population of voters vulnerable to the views of a few antiestablishment nodes may help guard populations from manipulation and fake information during elections and other scenarios.

\section{ACKNOWLEDGMENTS}

Part of this work was carried out at the Mathematical Institute at University of Oxford. We thank James Gleeson, Kameron Decker Harris, Shankar Iyer, and Mikko Kivelä for 
helpful discussions, and M.A.P. thanks Ben Williamson for early inspiration to study hipsters. We also thank Serge Galam for bringing his work to our attention. J.S.J. thanks the Mathematical Institute at University of Oxford for hospitality and
Mogens H. Jensen (Niels Bohr Institute, University of Copenhagen) for making this project possible; and he acknowledges funding through the University of Copenhagen UCPH 2016 Excellence Programme for Interdisciplinary Research.
[1] S. Lehmann and Y.-Y. Ahn (eds.), Complex Spreading Phenomena in Social Systems: Influence and Contagion in Real-World Social Networks (Springer International Publishing, Cham, Switzerland, 2018); preprints of some chapters are available at https://socialcontagionbook.github.io.

[2] M. A. Porter and J. P. Gleeson, Dynamical systems on networks: A tutorial, Front. Appl. Dyn. Syst.: Rev. Tutorials 4, 1 (2016).

[3] N. A. Christakis and J. H. Fowler, Social contagion theory: Examining dynamic social networks and human behavior, Stat. Med. 32, 556 (2013).

[4] J. Borge-Holthoefer, R. A. Baños, S. González-Bailón, and Y. Moreno, Cascading behavior in complex socio-technical networks, J. Complex Networks 1, 3 (2013).

[5] M. Granovetter, Threshold models of collective behavior, Am. J. Soc. 83, 1420 (1978).

[6] T. W. Valente, Network Models of the Diffusion of Innovations (Hampton Press, Creskill, NJ, USA, 1995).

[7] M. O. Jackson and D. López-Pintado, Diffusion and contagion in networks with heterogeneous agents and homophily, Network Sci. 1, 49 (2013).

[8] D. Kempe, J. Kleinberg, and É. Tardos, Maximizing the spread of influence through a social network, in Proceedings of the Ninth ACM SIGKDD International Conference on Knowledge Discovery and Data Mining, KDD '03 (ACM, New York, NY, USA, 2003), pp. 137-146.

[9] D. J. Watts, A simple model of global cascades on random networks, Proc. Natl. Acad. Sci. U.S.A. 99, 5766 (2002).

[10] C. Castellano, S. Fortunato, and V. Loreto, Statistical physics of social dynamics, Rev. Mod. Phys. 81, 591 (2009).

[11] N. A. Christakis and J. H. Fowler, The spread of obesity in a large social network over 32 years, New Engl. J. Med. 357, 370 (2007).

[12] D. Centola and M. W. Macy, Complex contagions and the weakness of long ties, Am. J. Soc. 113, 702 (2007).

[13] P. S. Dodds and D. J. Watts, A generalized model of social and biological contagion, J. Theor. Biol. 232, 587 (2005).

[14] S. Aral, L. Muchnik, and A. Sundararajan, Distinguishing influence-based contagion from homophily-driven diffusion in dynamic networks, Proc. Natl. Acad. Sci. U.S.A. 106, 21544 (2009).

[15] J. Ugander, L. Backstrom, C. Marlow, and J. Kleinberg, Structural diversity in social contagion, Proc. Natl. Acad. Sci. U.S.A. 109, 5962 (2012).

[16] S. Goel, A. Anderson, J. Hofman, and D. J. Watts, The structural virality of online diffusion, Manage. Sci. 62, 180 (2016).

[17] B. Mønsted, P. Sapieżyński, E. Ferrara, and S. Lehmann, Evidence of complex contagion of information in social media: An experiment using Twitter bots, PLoS One 12, e0184148 (2017).

[18] D. Mollison, Spatial contact models for ecological and epidemic spread, J. R. Stat. Soc.: Ser. B (Methodol.) 39, 283 (1977).
[19] H. P. Young, Innovation diffusion in heterogeneous populations: Contagion, social influence, and social learning, Am. Econ. Rev. 99, 1899 (2009).

[20] D. Centola, R. Willer, and M. W. Macy, The emperor's dilemma: A computational model of self-enforcing norms, Am. J. Soc. 110, 1009 (2005).

[21] H. Elsinger, A. Lehar, and M. Summer, Risk assessment for banking systems, Manage. Sci. 52, 1301 (2006).

[22] J. Juul and K. Sneppen, Locally self-organized quasicritical percolation in a multiple-disease model, Phys. Rev. E 84, 036119 (2011).

[23] P. S. Dodds and D. J. Watts, Universal Behavior in a Generalized Model of Contagion, Phys. Rev. Lett. 92, 218701 (2004).

[24] I. Z. Kiss, J. C. Miller, and P. L. Simon, Mathematics of Epidemics on Networks: From Exact to Approximate Models (Springer International Publishing, Cham, Switzerland, 2017).

[25] S. Melnik, J. A. Ward, J. P. Gleeson, and M. A. Porter, Multistage complex contagions, Chaos 23, 013124 (2013).

[26] F. J. Pérez-Reche, J. J. Ludlam, S. N. Taraskin, and C. A. Gilligan, Synergy in Spreading Processes: From Exploitative to Explorative Foraging Strategies, Phys. Rev. Lett. 106, 218701 (2011).

[27] J. S. Juul and M. A. Porter, Synergistic effects in threshold models on networks, Chaos 28, 013115 (2018).

[28] R. Pastor-Satorras, C. Castellano, P. Van Mieghem, and A. Vespignani, Epidemic processes in complex networks, Rev. Mod. Phys. 87, 925 (2015).

[29] D. Taylor, F. Klimm, H. A. Harrington, M. Kramár, K. Mischaikow, M. A. Porter, and P. J. Mucha, Topological data analysis of contagion maps for examining spreading processes on networks, Nat. Commun. 6, 7723 (2015).

[30] E. Valdano, L. Ferreri, C. Poletto, and V. Colizza, Analytical Computation of the Epidemic Threshold on Temporal Networks, Phys. Rev. X 5, 021005 (2015).

[31] J. P. Gleeson, Binary-State Dynamics on Complex Networks: Pair Approximation and Beyond, Phys. Rev. X 3, 021004 (2013).

[32] J. P. Gleeson, Cascades on correlated and modular random networks, Phys. Rev. E 77, 046117 (2008).

[33] T. R. Hurd and J. P. Gleeson, On Watts' cascade model with random link weights, J. Complex Networks 1, 25 (2013).

[34] M. Zheng, L. Lü, and M. Zhao, Spreading in online social networks: The role of social reinforcement, Phys. Rev. E 88, 012818 (2013).

[35] J. P. Gleeson, K. P. O’Sullivan, R. A. Baños, and Y. Moreno, Effects of Network Structure, Competition, and Memory Time on Social Spreading Phenomena, Phys. Rev. X 6, 021019 (2016).

[36] X. Qiu, D. F. M. Oliveira, A. S. Shirazi, A. Flammini, and F. Menczer, Limited individual attention and online virality of low-quality information, Nat. Hum. Behav. 1, 0132 (2017). 
[37] J. Chalupa, P. L. Leath, and G. R. Reich, Bootstrap percolation on a Bethe lattice, J. Phys. C: Solid State Phys. 12, L31 (1979).

[38] D. Centola, V. M. Eguíluz, and M. W. Macy, Cascade dynamics of complex propagation, Physica A 374, 449 (2007).

[39] S.-W. Oh and M. A. Porter, Complex contagions with timers, Chaos 28, 033101 (2018).

[40] E. M. Rogers, Diffusion of Innovations (Simon and Schuster, New York, NY, USA, 2010).

[41] F. M. Bass, A new product growth for model consumer durables, Manage. Sci. 15, 215 (1969).

[42] F. M. Bass, The relationship between diffusion rates, experience curves, and demand elasticities for consumer durable technological innovations, J. Bus. 53, S51 (1980).

[43] J. V. Nevers, Extensions of a new product growth model, Sloan Manage. Rev. 13, 77 (1972).

[44] F. M. Bass, T. V. Krishnan, and D. C. Jain, Why the Bass model fits without decision variables, Marketing Sci. 13, 203 (1994).

[45] F. M. Bass, Comments on "A new product growth for model consumer durables: The Bass model", Manage. Sci. 50, 1833 (2004).

[46] M. B. Gordon, M. F. Laguna, S. Gonçalves, and J. R. Iglesias, Adoption of innovations with contrarian agents and repentance, Physica A 486, 192 (2017).

[47] P. S. Dodds, K. D. Harris, and C. M. Danforth, Limited Imitation Contagion on Random Networks: Chaos, Universality, and Unpredictability, Phys. Rev. Lett. 110, 158701 (2013).

[48] K. D. Harris, C. M. Danforth, and P. S. Dodds, Dynamical influence processes on networks: General theory and applications to social contagion, Phys. Rev. E 88, 022816 (2013).

[49] S. Galam, Majority rule, hierarchical structures, and democratic totalitarianism: A statistical approach, J. Math. Psychol. 30, 426 (1986).

[50] S. Galam, Contrarian deterministic effects on opinion dynamics: The hung elections scenario, Physica A 333, 453 (2004).

[51] S. Galam and F. Jacobs, The role of inflexible minorities in the breaking of democratic opinion dynamics, Physica A 381, 366 (2007).

[52] S. Galam, Stubbornness as an unfortunate key to win a public debate: An illustration from sociophysics, Mind Society 15, 117 (2016).

[53] P. Nyczka, K. Sznajd-Weron, and J. Cisło, Phase transitions in the $q$-voter model with two types of stochastic driving, Phys. Rev. E 86, 011105 (2012).

[54] P. Nyczka and K. Sznajd-Weron, Anticonformity or independence? Insights from statistical physics, J. Stat. Phys. 151, 174 (2013).

[55] N. Khalil and R. Toral, The noisy voter model under the influence of contrarians, Physica A 515, 81 (2019).

[56] R. Apriasz, T. Krueger, G. Marcjasz, and K. Sznajd-Weron, The hunt opinion model-An agent based approach to recurring fashion cycles, PLoS One 11, e0166323 (2016).

[57] K. Sznajd-Weron and R. Weron, How effective is advertising in duopoly markets? Physica A 324, 437 (2003).

[58] A. Mellor, M. Mobilia, S. Redner, A. M. Rucklidge, and J. A. Ward, Influence of Luddism on innovation diffusion, Phys. Rev. E 92, 012806 (2015).
[59] J. P. Gambaro and N. Crokidakis, The influence of contrarians in the dynamics of opinion formation, Physica A 486, 465 (2017).

[60] E. Ferrara, Disinformation and social bot operations in the run up to the 2017 French presidential election, First Monday 22, 8 (2017).

[61] A. Bessi and E. Ferrara, Social bots distort the 2016 US Presidential election online discussion, First Monday 21, 11 (2016).

[62] D. Guilbeault, J. Becker, and D. Centola, Social learning and partisan bias in the interpretation of climate trends, Proc. Natl. Acad. Sci. U.S.A. 115, 9714 (2018).

[63] K. H. Jamieson and B. W. Hardy, Leveraging scientific credibility about Arctic Sea ice trends in a polarized political environment, Proc. Natl. Acad. Sci. U.S.A. 111, 13598 (2014).

[64] Michael Bang Petersen, Mathias Osmundsen, and Kevin Arceneaux, A "Need for Chaos" and the Sharing of Hostile Political Rumors in Advanced Democracies (2018), PsyArXiv, https: //doi.org/10.31234/osf.io/6m4ts.

[65] S. B. Hobolt, The Brexit vote: A divided nation, a divided continent, J. Eur. Public Policy 23, 1259 (2016).

[66] J. E. Oliver and W. M. Rahn, Rise of the Trumpenvolk: Populism in the 2016 election, Ann. Am. Acad. Political Social Sci. 667, 189 (2016).

[67] J. Touboul, The hipster effect: When anticonformists all look the same, arXiv: 1410.8001.

[68] J. P. Gleeson, D. Cellai, J.-P. Onnela, M. A. Porter, and F. Reed-Tsochas, A simple generative model of collective online behavior, Proc. Natl. Acad. Sci. U.S.A. 111, 10411 (2014).

[69] A. L. Traud, P. J. Mucha, and M. A. Porter, Social structure of Facebook networks, Physica A 391, 4165 (2012).

[70] J. P. Gleeson and D. J. Cahalane, Seed size strongly affects cascades on random networks, Phys. Rev. E 75, 056103 (2007).

[71] S. Melnik, A. Hackett, M. A. Porter, P. J. Mucha, and J. P. Gleeson, The unreasonable effectiveness of tree-based theory for networks with clustering, Phys. Rev. E 83, 036112 (2011).

[72] Pair approximations have also been employed in other types of opinion models, such as for a $q$-voter model in [78].

[73] S. Goel, D. J. Watts, and D. G. Goldstein, The structure of online diffusion networks, in Proceedings of the 13th ACM Conference on Electronic Commerce, EC '12 (ACM, New York, NY, USA, 2012), doi:10.1145/2229012.2229058.

[74] B. K. Fosdick, D. B. Larremore, J. Nishimura, and J. Ugander, Configuring random graph models with fixed degree sequences, SIAM Rev. 60, 315 (2018).

[75] M. E. J. Newman, Networks, 2nd ed. (Oxford University Press, Oxford, UK, 2018).

[76] The original hipster was in the network before it was popular.

[77] P. G. Fennell, S. Melnik, and J. P. Gleeson, Limitations of discrete-time approaches to continuous-time contagion dynamics, Phys. Rev. E 94, 052125 (2016).

[78] A. Jedrzejewski, Pair approximation for the $q$-voter model with independence on complex networks, Phys. Rev. E 95, 012307 (2017).

[79] Our code is available at https://sid.erda.dk/share_redirect/ hZOCeo4qU5. 